\title{
Improved Security for a Ring-Based Fully Homomorphic Encryption Scheme
}

\author{
Joppe W. Bos ${ }^{1}$, Kristin Lauter ${ }^{1}$, Jake Loftus $^{2 \star}$, and Michael Naehrig ${ }^{1}$ \\ 1 Microsoft Research, \{jbos, klauter, mnaehrig\}@microsoft.com \\ 2 University of Bristol, loftus@cs.bris.ac.uk
}

\begin{abstract}
In 1996, Hoffstein, Pipher and Silverman introduced an efficient lattice based encryption scheme dubbed NTRUEncrypt. Unfortunately, this scheme lacks a proof of security. However, in 2011, Stehlé and Steinfeld showed how to modify NTRUEncrypt to reduce security to standard problems in ideal lattices. In 2012, López-Alt, Tromer and Vaikuntanathan proposed a fully homomorphic scheme based on this modified system. However, to allow homomorphic operations and prove security, a non-standard assumption is required. In this paper, we show how to remove this non-standard assumption via techniques introduced by Brakerski and construct a new fully homomorphic encryption scheme from the Stehlé and Steinfeld version based on standard lattice assumptions and a circular security assumption. The scheme is scale-invariant and therefore avoids modulus switching and the size of ciphertexts is one ring element. Moreover, we present a practical variant of our scheme, which is secure under stronger assumptions, along with parameter recommendations and promising implementation results. Finally, we present an approach for encrypting larger input sizes by extending ciphertexts to several ring elements via the CRT on the message space.
\end{abstract}

\section{Introduction}

Fully homomorphic encryption (FHE) is a powerful form of encryption which allows an untrusted server to carry out arbitrary computation on encrypted data on behalf of a client. Introduced in [20] by Adleman, Dertouzos and Rivest, the problem of constructing a scheme which can evaluate any function on encrypted data remained open until 2009, when Gentry constructed an FHE scheme based on ideal lattices [9]. Gentry's scheme effectively laid down a blueprint for constructing FHE schemes and paved the way for many further constructions $[26,2,3,5,4,23,19,10,8]$. The main focus of the cryptologic research community has been on improving the efficiency of FHE and basing its security on standard assumptions.

Recently, López-Alt et al. [15] proposed a (multi-key) FHE scheme based on the work by Stehlé and Steinfeld [24] in which a provably secure version of

* Most of this work was done while the third author was an intern in the Cryptography Research group at Microsoft Research. 
NTRUEncrypt [12] is presented with security based on standard problems in ideal lattices. Unfortunately, the FHE scheme from [15] needs to make an additional assumption relating to the uniformity of the public key, the so-called decisional small polynomial ratio (DSPR) assumption, to allow homomorphic operations and remain semantically secure. We show how to avoid this additional assumption and transform the results from [24] into a fully homomorphic encryption scheme based on standard lattice assumptions only. This is achieved by limiting noise growth during homomorphic operations via a tensoring technique recently introduced by Brakerski [2]. Besides this theoretical advantage, our scheme has other attractive properties. Firstly, this new scheme is scale-invariant in the sense of [2], i.e. it avoids the modulus-switching technique of Brakerski, Gentry and Vaikuntanathan [3]. Secondly, we keep the property of the scheme in [15] that a ciphertext consists of only a single ring element as opposed to the two or more ring elements for schemes based purely on the (ring) learning with errors (RLWE) assumption [16]. This decreases the ciphertext size since parameters are comparable in both settings. Finally, we present a technique to increase the size of the input space by working with separate, small plaintext moduli in ciphertexts of multiple ring elements, which are later combined via the Chinese remainder theorem into a larger plaintext modulus. For some applications, this additional flexibility to increase the message space without changing parameters at the cost of increasing ciphertext size can prove especially useful.

Our main contribution is an FHE scheme based on the schemes by Stehlé and Steinfeld [24] and López-Alt et al. [15] that does not need the DSPR assumption and thus is secure under the RLWE and circular security assumptions only. The public key in both schemes is the fraction $h=g f^{-1} \bmod q$ of two polynomials $f$ and $g$ in a cyclotomic polynomial ring modulo an integer modulus $q$ that are sampled from a discrete Gaussian distribution. The DSPR assumption is the assumption that such a fraction is indistinguishable from uniform random in the ring modulo $q$. Stehle and Steinfeld show that this assumption holds if the Gaussian is wide enough. Unfortunately, the scheme by López-Alt et al. cannot use such a wide Gaussian for key generation. Since the norms of $f$ and $g$ contribute to the noise growth during homomorphic multiplication, using a wide enough Gaussian means that the scheme is not guaranteed to be capable of doing even a single multiplication. We solve this problem by using decompositions and Brakerski's [2] tensoring technique. During the homomorphic multiplication procedure which includes a key switching step, we decompose the polynomial $f$ into its bit decomposition, i.e. into a vector of polynomials with binary coefficients. This technique replaces the ring product of polynomials by a scalar product of binary decomposition vectors with vectors of polynomials multiplied by powers of 2 modulo $q$. The noise growth introduced in such a scalar product is bounded by a polynomial in $\log (q)$ and the degree of $f$, replacing the square of the norm of $f$ in the bounds of the original scheme. Noise growth is much smaller now and it is possible to sample from a wide Gaussian to ensure the Stehlé-Steinfeld conditions. 
As noted in Appendix A.1 of [15], any FHE scheme is inherently a multikey scheme for a constant number of parties, but this construction is rather inefficient. The original scheme in [15], however, directly yields the multi-key property for a non-constant number of parties, which is much more efficient. Our scheme is not a multi-key scheme in that sense because decryption of a multi-key ciphertext would require a multiplication by the product of all keys that were involved in the generation of the ciphertext. With keys generated in the setting of Stehlé and Steinfeld, multiplying by a product of only two keys would already lead to a noise overflow, making it impossible to decrypt correctly.

The second part of the paper describes a more practical variant of the above scheme, along with details on parameter selection and implementation results. The price for obtaining security without the DSPR assumption in the above scheme lies in a large evaluation key and a complicated key switching procedure, both of which are a consequence of using the tensoring approach. Any possibility, which we are aware of, to avoid the tensor products, leads to an increase in the noise bounds that makes it necessary to reintroduce the DSPR assumption. However, if one is willing to make this assumption, there are several efficiency advantages and possible trade-offs as shown in our more practical variant. This variant keeps the general characteristics of the scheme, but simplifies key switching and avoids tensor products. A much shorter evaluation key can be achieved by using base- $w$ instead of base- 2 decompositions for a $w>2$, e.g. $w=2^{32}$. This increases noise growth, but ensures that the evaluation key contains only a few ring elements. Since the key switching is the main cost in homomorphic multiplication, the choice of $w$ provides an important trade-off between homomorphic capability and multiplication efficiency. We also point out that it is possible to weaken the DSPR assumption by allowing the polynomial $g$ to be sampled from a wider Gaussian than $f$.

This paper is organized as follows. In Section 2 we recall basic mathematical techniques used throughout the paper, as well as the RLWE and DSPR assumptions. Section 3 states the public-key encryption scheme that is the foundation for the new leveled homomorphic scheme introduced in Section 4. This section also discusses correctness and security, and shows that the leveled homomorphic scheme can be bootstrapped to a fully homomorphic scheme. A more practical variant of the leveled scheme is introduced in Section 5 together with its security analysis, recommendations for secure parameters, and implementation performance numbers. We also present some optimizations including the CRT approach. Section 6 concludes the paper. The proofs of most lemmas and theorems are given in respective appendices.

\section{Preliminaries}

In this section, we define all basic notation that is needed in the paper. The most important structure is the ring $R$. Let $d$ be a positive integer and define $R=$ $\mathbb{Z}[X] /\left(\Phi_{d}(X)\right)$ as the ring of polynomials with integer coefficients modulo the $d$ th cyclotomic polynomial $\Phi_{d}(X) \in \mathbb{Z}[X]$. The degree of $\Phi_{d}$ is $n=\varphi(d)$, where $\varphi$ 
is Euler's totient function. The elements of $R$ can be uniquely represented by all polynomials in $\mathbb{Z}[X]$ of degree less than $n$. Arithmetic in $R$ is arithmetic modulo $\Phi_{d}(X)$, which is implicit whenever we write down terms or equalities involving elements in $R$. An arbitrary element $a \in R$ can be written as $a=\sum_{i=0}^{n-1} a_{i} X^{i}$ with $a_{i} \in \mathbb{Z}$ and we identify $a$ with its vector of coefficients $\left(a_{0}, a_{1}, \ldots, a_{n-1}\right)$. In particular, $a$ can be viewed as an element of the $\mathbb{R}$-vector space $\mathbb{R}^{n}$. We choose the maximum norm on $\mathbb{R}^{n}$ to measure the size of elements in $R$. The maximum norm of $a$ is defined as $\|a\|_{\infty}=\max _{i}\left\{\left|a_{i}\right|\right\}$.

When multiplying two elements $g, h \in R$, the norm of their product $g h$ expands with respect to the individual norms of $g$ and $h$. The maximal norm expansion that can occur is $\delta=\sup \left\{\|g \cdot h\|_{\infty} /\left(\|g\|_{\infty}\|h\|_{\infty}\right): g, h \in R\right\}$, which is a ring constant. When $d$ is a power of 2 and thus $\Phi_{d}(X)=X^{n}+1$, we have $\delta=n[9$, Section 3.4]. To keep the exposition more general, we do not restrict to this special case and work with general $\delta$ in most of what follows.

Let $\chi$ be a probability distribution on $R$. We assume that we can efficiently sample elements from $R$ according to $\chi$, and we use the standard notation $a \leftarrow \chi$ to denote that $a \in R$ is sampled from $\chi$. The distribution $\chi$ on $R$ is called $B$ bounded for some $B>0$ if for all $a \leftarrow \chi$ we have $\|a\|_{\infty}<B$, i.e. $a$ is $B$-bounded (see [3, Def. 3] and [15, Def. 3.1 and 3.2]). Let us introduce a specific example of a distribution on $R$. First, define the discrete Gaussian distribution $D_{\mathbb{Z}, \sigma}$ with mean 0 and standard deviation $\sigma$ over the integers, which assigns a probability proportional to $\exp \left(-\pi|x|^{2} / \sigma^{2}\right)$ to each $x \in \mathbb{Z}$. When $d$ is a power of 2 and $\Phi_{d}(X)=X^{n}+1$, we can take $\chi$ to be the spherical discrete Gaussian $\chi=\mathcal{D}_{\mathbb{Z}^{n}, \sigma}$, where each coefficient of the polynomial is sampled according to the one-dimensional distribution $D_{\mathbb{Z}, \sigma}$ (see [16] for more details and why $\chi=\mathcal{D}_{\mathbb{Z}^{n}, \sigma}$ is the right choice in that case). The distribution $\chi$ is used in many fully homomorphic encryption schemes based on RLWE to sample random error polynomials that have small coefficients with high probability. Such polynomials are a significant part of the noise terms used in the encryption process. To deduce meaningful bounds on noise size and noise growth during homomorphic operations, we assume that the distribution we are working with is $B$-bounded for some $B$. For the discrete Gaussian, this is a reasonable assumption since sampled elements tend to be small with high probability. By rejecting samples with norm larger than $B$, we can sample from a truncated Gaussian distribution that is statistically close to the true discrete Gaussian if $B$ is chosen large enough. For example, if we take $B=6 \sigma$, all samples are $B$-bounded with very high probability [17, Lemma 4.4].

Although the principal object of interest for our scheme is the ring $R$, and all polynomials that we deal with are considered to be elements of $R$, we often reduce polynomial coefficients modulo an integer modulus $q$. We denote the map that reduces an integer $x$ modulo $q$ and uniquely represents the result by an element in the interval $(-q / 2, q / 2]$ by $[\cdot]_{q}$. We extend this map to polynomials in $\mathbb{Z}[X]$ and thus also to elements of $R$ by applying it to their coefficients separately, i.e. $[\cdot]_{q}: R \rightarrow R, a=\sum_{i=0}^{n-1} a_{i} X^{i} \mapsto \sum_{i=0}^{n-1}\left[a_{i}\right]_{q} X^{i}$. Furthermore, we extend this notation to vectors of polynomials by applying it to the entries of the vectors 
separately. Sometimes we reduce an integer modulo $q$ and uniquely represent the result by an element in $[0, q)$. In this case, we write $r_{q}(x)$ to mean the reduction of $x$ into $[0, q)$. A polynomial $f \in R$ is invertible modulo $q$ if there exists a polynomial $f^{-1} \in R$ such that $f f^{-1}=\tilde{f}$, where $\tilde{f}(X)=\sum_{i} a_{i} X^{i}$ with $a_{0}=1$ $\bmod q$ and $a_{j}=0 \bmod q$ for all $j \neq 0$. Our homomorphic encryption scheme uses two different moduli. In addition to a modulus $q$ that is used to reduce the coefficients of the elements that represent ciphertexts, there is a second modulus $t<q$ that determines the message space $R / t R$, i.e. messages are polynomials in $R$ modulo $t$. We make frequent use of the quantity $\Delta=\lfloor q / t\rfloor$ and it is readily verified that $q-r_{t}(q)=\Delta \cdot t$.

In [2], functions called BitDecomp and PowersOfTwo are used. We slightly generalize these to an arbitrary base and describe our notation next. Fix a positive integer $w>1$ that is used to represent integers in a radix- $w$ system. Let $\ell_{w, q}=\left\lfloor\log _{w}(q)\right\rfloor+2$, then a non-negative integer $z<q$ can be written as $\sum_{i=0}^{\ell_{w, q}-2} z_{i} w^{i}$ where the $z_{i}$ are integers such that $0 \leq z_{i}<w$. If $z$ is an integer in the interval $(-q / 2, q / 2]$, it can be written uniquely as $\sum_{i=0}^{\ell_{w, q}-1} z_{i} w^{i}$ with $z_{i} \in(-w / 2, w / 2]$. With this, an element $x \in R$ with coefficients in $(-q / 2, q / 2]$ can be written as $\sum_{i=0}^{\ell_{w, q}-1} x_{i} w^{i}$, where $x_{i} \in R$ with coefficients in $(-w / 2, w / 2]$. Since then $x_{i}=\left[x_{i}\right]_{w}$, we write $x=\sum_{i=0}^{\ell_{w, q}-1}\left[x_{i}\right]_{w} w^{i}$ to make clear that the norm of the coefficient polynomials $x_{i}$ is at most $w / 2$. With this notation, define

$$
D_{w, q}: R \rightarrow R^{\ell_{w, q}}, \quad x \mapsto\left(\left[x_{0}\right]_{w},\left[x_{1}\right]_{w}, \ldots,\left[x_{\ell_{w, q}-1}\right]_{w}\right)=\left(\left[x_{i}\right]_{w}\right)_{i=0}^{\ell_{w, q}-1},
$$

this function for $w=2$ is called BitDecomp in [2]. We define a second function

$$
P_{w, q}: R \rightarrow R^{\ell_{w, q}}, \quad x \mapsto\left([x]_{q},[x w]_{q}, \ldots,\left[x w^{\ell_{w, q}-1}\right]_{q}\right)=\left(\left[x w^{i}\right]_{q}\right)_{i=0}^{\ell_{w, q}-1},
$$

which is called PowersOfTwo in [2] for $w=2$. For any two $x, y \in R$, we see that the scalar product of the vectors $D_{w, q}(x)$ and $P_{w, q}(y)$ is the same as the product $x y$ modulo $q$, because

$$
\left\langle D_{w, q}(x), P_{w, q}(y)\right\rangle=\sum_{i=0}^{\ell_{w, q}-1}\left[x_{i}\right]_{w}\left[y w^{i}\right]_{q} \equiv y \sum_{i=0}^{\ell_{w, q}-1}\left[x_{i}\right]_{w} w^{i} \equiv x y \quad(\bmod q) .
$$

Note that when $\|f\|_{\infty}<B$ for some $B<q$, then only the $\ell_{w, B}:=\left\lfloor\log _{w}(B)\right\rfloor+2$ least significant polynomials in $D_{w, q}(f)$ can be non-zero. We use the tensor product of two vectors in the usual way, i.e. for a positive integer $\ell$ and two vectors $a, b \in R^{\ell}$, the tensor $a \otimes b \in R^{\ell^{2}}$ is the concatenation of the $a_{i} b$ for $i \in\{1,2, \ldots, \ell\}$. We extend the functions $D_{w, q}$ and $P_{w, q}$ to vectors. For $v=$ $\left(v_{1}, v_{2}, \ldots, v_{\ell}\right) \in R^{\ell}$ denote the vector $\left(D_{w, q}\left(v_{1}\right), \ldots, D_{w, q}\left(v_{\ell}\right)\right) \in R^{\ell \cdot \ell_{w, q}}$ by $D_{w, q}(v)$, likewise we extend $P_{w, q}$.

Several operations in the scheme require scaling by rational numbers such that the resulting polynomials do not necessarily belong to $R$ but instead have rational coefficients. In that case, a rounding procedure is applied to get back to integer coefficients. The usual rounding of a rational number $a$ to the nearest integer is denoted by $\lfloor a\rceil$. 
The Ring Learning With Errors (RLWE) Problem. Our scheme relies on the hardness of the (decisional) ring learning with errors problem, which was first introduced by Lyubashevsky, Peikert and Regev [16].

Definition 1 (Decision-RLWE). Given a security parameter $\lambda$, let $d$ and $q$ be integers depending on $\lambda$, let $R=\mathbb{Z}[X] /\left(\Phi_{d}(X)\right)$ and let $R_{q}=R / q R$. Given a distribution $\chi$ over $R_{q}$ that depends on $\lambda$, the Decision- $\mathrm{RLWE}_{d, q, \chi}$ problem is to distinguish the following two distributions. The first distribution consists of pairs $(a, u)$, where $a, u \leftarrow R_{q}$ are drawn uniformly at random from $R_{q}$. The second distribution consists of pairs of the form $(a, a \cdot s+e)$. The element $s \leftarrow R_{q}$ is drawn uniformly at random and is fixed for all samples. For each sample, $a \leftarrow R_{q}$ is drawn uniformly at random, and $e \leftarrow \chi$. The Decision- $\mathrm{RLWE}_{d, q, \chi}$ assumption is that the Decision- $\mathrm{RLWE}_{d, q, \chi}$ problem is hard.

In [16], it was shown that the hardness of RLWE can be established by a quantum reduction to worst-case shortest vector problems in ideal lattices over the ring $R$, see also [3, Thm. 2]. It is known that the search variant of $\operatorname{RLWE}_{d, q, \chi}$, in which we are required to explicitly find the secret $s$ given an $\operatorname{RLWE}_{d, q, \chi}$ instance, is equivalent to the decision problem [16]. There are a number of variants of RLWE which are as hard as RLWE, for example we can restrict the sampling of $a$ and $e$ to invertible elements only [24]. And we can also choose $s$ from $\chi$ without incurring any loss of security [1].

The Decisional Small Polynomial Ratio (DSPR) Problem. In [15], LópezAlt, Tromer and Vaikuntanathan introduced the decisional small polynomial ratio problem. They describe a multi-key fully homomorphic encryption scheme with security based on the assumption that the DSPR problem is hard in the $\operatorname{ring} R_{q}$ where $R=\mathbb{Z}[x] /\left(x^{n}+1\right)$ for $n$ a power of 2 and $t=2$. We state a more general form of the problem for any cyclotomic ring $R=\mathbb{Z}[x] /\left(\Phi_{d}(x)\right)$ and general $1<t<q$. Let $h=t g / f(\bmod q)$ where $f=1+t f^{\prime}$ and $f^{\prime}, g \leftarrow \chi$ where $\chi$ is a truncated Gaussian distribution. In [15], the problem of distinguishing such an element $h$ from a uniformly random element of $R_{q}=R / q R$ was formalized as the DSPR problem. Assuming the hardness of DSPR and RLWE, the scheme in [15] is secure. To state the problem, define the following: for a distribution $\chi$ on $R_{q}$ and $z \in R_{q}$ we define $\chi_{z}=\chi+z$ to be the distribution shifted by $z$. Also, let $R_{q}^{\times}$be the set of all invertible elements in $R_{q}$.

Definition 2 (DSPR). For security parameter $\lambda$, let $d$ and $q$ be integers, let $R=\mathbb{Z}[X] /\left(\Phi_{d}(X)\right)$ and $R_{q}=R / q R$ and let $\chi$ be a distribution over $R_{q}$, all depending on $\lambda$. Let $t \in R_{q}^{\times}$be invertible in $R_{q}, y_{i} \in R_{q}$ and $z_{i}=-y_{i} t^{-1}$ $(\bmod q)$ for $i \in\{1,2\}$. The $\mathrm{DSPR}_{d, q, \chi}$ problem is to distinguish elements of the form $h=a / b$ where $a \leftarrow y_{1}+t \cdot \chi_{z_{1}}, b \leftarrow y_{2}+t \cdot \chi_{z_{2}}$ from uniformly random elements of $R_{q}$. The $\mathrm{DSPR}_{d, q, \chi}$ assumption is that the $\mathrm{DSPR}_{d, q, \chi}$ problem is hard.

Theorem 4.1 in the full version of [24] shows that $\operatorname{DSPR}_{d, q, \chi}$ is hard when the $\chi_{z_{i}}$ are shifted versions of a discrete Gaussian distributions $\chi$ which is $\mathcal{D}_{\mathbb{Z}^{n}, \sigma}$ restricted to $R_{q}^{\times}$for a large enough deviation $\sigma$. For convenience, we state the theorem in Appendix A. A discrete Gaussian on $R_{q}^{\times}$can be obtained from a discrete Gaussian on $R_{q}$ by rejecting non-invertible elements. 


\section{Basic Scheme}

In this section, we describe the basic public key encryption scheme that is the foundation for the leveled schemes of the next sections. The scheme is parameterized by a modulus $q$ and a plaintext modulus $1<t<q$. Ciphertexts are elements of $R=\mathbb{Z}[X] /\left(\Phi_{d}(X)\right)$ and plaintexts are elements of $R / t R$ (see Section 2 ). Secret keys and errors are generated from different distributions, for example Gaussian distributions of different width. The secret key is derived from the distribution $\chi_{\text {key }}$, and errors are sampled from the distribution $\chi_{\text {err }}$. We use "Regev-style" encryption as in [2] and [8]. The scheme consists of the following algorithms.

- Basic.ParamsGen $(\lambda)$ : Given the security parameter $\lambda$, fix a positive integer $d$ that determines $R$, moduli $q$ and $t$ with $1<t<q$, and distributions $\chi_{\text {key }}, \chi_{\text {err }}$ on $R$. Output $\left(d, q, t, \chi_{\text {key }}, \chi_{\text {err }}\right)$.

- Basic.KeyGen $\left(d, q, t, \chi_{\mathrm{key}}, \chi_{\mathrm{err}}\right)$ : Sample $f^{\prime}, g \leftarrow \chi_{\mathrm{key}}$ and let $f=\left[t f^{\prime}+1\right]_{q}$. If $f$ is not invertible modulo $q$, choose a new $f^{\prime}$. Compute the inverse $f^{-1} \in R$ of $f$ modulo $q$ and set $h=\left[t g f^{-1}\right]_{q}$. Output the public and private key pair $(\mathrm{pk}, \mathrm{sk})=(h, f) \in R^{2}$.

- Basic.Encrypt $(h, m)$ : The message space is $R / t R$. For a message $m+t R$, choose $[m]_{t}$ as its representative. Sample $s, e \leftarrow \chi_{\text {err }}$, and output the ciphertext

$$
c=\left[\lfloor q / t\rfloor[m]_{t}+e+h s\right]_{q} \in R .
$$

- Basic.Decrypt $(f, c)$ : To decrypt a ciphertext $c$, compute

$$
m=\left[\left\lfloor\frac{t}{q} \cdot[f c]_{q}\right]\right]_{t} \in R .
$$

In the following, we often refer to a message as an element $m$ in the ring $R$ although the message space is $R / t R$, keeping in mind that encryption always takes place on the representative $[m]_{t}$ and that by decrypting, all that can be recovered is $m$ modulo $t$.

Correctness. The following lemma states conditions for a ciphertext $c$ such that the decryption algorithm outputs the message $m$ that was originally encrypted.

Lemma 1. Let $q$, $t$, and $\Delta=\lfloor q / t\rfloor$ be as above and let $c, f, m \in R$. If there exists $v \in R$ such that

$$
f c=\Delta[m]_{t}+v \quad(\bmod q) \text { and }\|v\|_{\infty}<\left(\Delta-r_{t}(q)\right) / 2,
$$

then Basic.Decrypt $(f, c)=[m]_{t}$, i.e. $c$ decrypts correctly under the secret key $f$.

Of course, for any given $c, f$ and $m$, there always exists a $v \in R$ such that $f c=\Delta[m]_{t}+v(\bmod q)$. But only a $v$ of small norm allows one to recover $[m]_{t}$ from $c$. Since we are always free to vary $v$ modulo $q$, i.e. to add any multiple of $q$ to it, we choose $v$ to be the canonical element $[v]_{q}$. This means that we choose $v$ with the smallest possible norm among all polynomials that satisfy the 
equation. We call this specific $v$ the inherent noise in $c$ with respect to $m$ and $f$. The previous lemma says that if the inherent noise in a ciphertext is small enough, then decryption works correctly.

Inherent noise in initial ciphertexts. The following lemma derives a bound on the inherent noise in a freshly encrypted ciphertext output by Basic.Encrypt, assuming bounds $B_{\text {key }}$ on the key and $B_{\text {err }}$ on the error distributions. Note that since $f^{\prime}, g \leftarrow \chi_{\text {key we have }}\left\|f^{\prime}\right\|_{\infty},\|g\|_{\infty}<B_{\text {key }}$ and it follows that $\|t g\|_{\infty}<t B_{\text {key }}$ and $\|f\|_{\infty}=\left\|1+t f^{\prime}\right\|_{\infty}<t B_{\text {key }}$ since $t \geq 2$.

Lemma 2. Let the key and error distributions be $B_{\mathrm{key}}$-bounded and $B_{\mathrm{err}}$-bounded, respectively. Given $m \in R$, a public key $h=\left[t g f^{-1}\right]_{q} \in R$ with secret key $f=\left[1+t f^{\prime}\right]_{q}, f^{\prime}, g \leftarrow \chi_{\mathrm{key}}$, and let $c=\operatorname{Basic} \operatorname{Encrypt}(h, m)$. There exists $v \in R$ such that $f c=\Delta[m]_{t}+v(\bmod q)$ and

$$
\|v\|_{\infty}<\delta t B_{\text {key }}\left(2 B_{\text {err }}+\frac{1}{2} r_{t}(q)\right) .
$$

In particular, by Lemma 1, decryption works correctly if $2 \delta t B_{\mathrm{key}}\left(2 B_{\mathrm{err}}+\frac{1}{2} r_{t}(q)\right)+$ $r_{t}(q)<\Delta$.

\section{Leveled Homomorphic Scheme}

In this section, we state our leveled homomorphic encryption scheme YASHE ${ }^{3}$ based on the Basic scheme from the previous section. We then analyze the homomorphic operations and deduce bounds on the noise growth that occurs during these operations.

- YASHE.ParamsGen $(\lambda)$ : Given the security parameter $\lambda$, output the parameters $\left(d, q, t, \chi_{\mathrm{key}}, \chi_{\mathrm{err}}, w\right)$, where $\left(d, q, t, \chi_{\mathrm{key}}, \chi_{\mathrm{err}}\right) \leftarrow \operatorname{BasicParamsGen}(\lambda)$ and $w>1$ is an integer.

- YASHE.KeyGen $\left(d, q, t, \chi_{\mathrm{key}}, \chi_{\mathrm{err}}, w\right)$ : Compute

$$
h, f \leftarrow \text { Basic.KeyGen }\left(d, q, t, \chi_{\mathrm{key}}, \chi_{\mathrm{err}}\right) .
$$

Sample $\boldsymbol{e}, s \leftarrow \chi_{\mathrm{err}}^{\ell_{w, q}^{3}}$, compute

$$
\gamma=\left[f^{-1} P_{w, q}\left(D_{w, q}(f) \otimes D_{w, q}(f)\right)+\boldsymbol{e}+h \cdot s\right]_{q} \in R^{\ell_{w, q}^{3}},
$$

and output (pk, sk, evk) $=(h, f, \gamma)$.

- YASHE.Encrypt(pk, $m)$ : To encrypt $m \in R$ output

$$
c \leftarrow \text { Basic.Encrypt }(\mathrm{pk}, m) \in R .
$$

- YASHE.Decrypt(sk, $c)$ : Output the message $m \leftarrow$ Basic.Decrypt(sk, $c) \in R$.

- YASHE.KeySwitch $\left(\tilde{c}_{\text {mult }}\right.$, evk $)$ : Output $\left[\left\langle D_{w, q}\left(\tilde{c}_{\text {mult }}\right), \text { evk }\right\rangle\right]_{q} \in R$.

\footnotetext{
${ }^{3}$ Yet Another Somewhat Homomorphic Encryption scheme.
} 
- YASHE.Add $\left(c_{1}, c_{2}\right)$ : Compute the addition of $c_{1}$ and $c_{2}$ as $c_{\text {add }}=\left[c_{1}+c_{2}\right]_{q}$.

- YASHE.Mult $\left(c_{1}, c_{2}\right.$, evk): Compute

$$
\tilde{c}_{\text {mult }}=\left[\left\lfloor\frac{t}{q} P_{w, q}\left(c_{1}\right) \otimes P_{w, q}\left(c_{2}\right)\right\rceil\right]_{q} \in R^{\ell_{w, q}^{2}},
$$

and output

$$
c_{\text {mult }}=\text { YASHE.KeySwitch }\left(\tilde{c}_{\text {mult }}, \text { evk }\right) .
$$

Since encryption and decryption are the same as in the Basic scheme from Section 3, the correctness bound does not change and Lemmas 1 and 2 hold for YASHE as well. Next, we analyze the homomorphic operations YASHE.Add and YASHE.Mult.

Homomorphic Addition. Given two ciphertexts $c_{1}, c_{2} \in R$, which encrypt two messages $m_{1}, m_{2}$ with inherent noise terms $v_{1}, v_{2}$, their sum modulo $q, c_{\text {add }}=$ $\left[c_{1}+c_{2}\right]_{q}$, encrypts the sum of the messages modulo $t,\left[m_{1}+m_{2}\right]_{t}$. Indeed, we can write $\left[m_{1}\right]_{t}+\left[m_{2}\right]_{t}=\left[m_{1}+m_{2}\right]_{t}+t r_{\text {add }}$ for some $r_{\text {add }} \in R$ with $\left\|r_{\text {add }}\right\|_{\infty} \leq 1$. Since

$$
\begin{aligned}
f\left[c_{1}+c_{2}\right]_{q}=f c_{1}+f c_{2} & =\Delta\left(\left[m_{1}\right]_{t}+\left[m_{2}\right]_{t}\right)+\left(v_{1}+v_{2}\right) \\
& =\Delta\left(\left[m_{1}+m_{2}\right]_{t}+t r_{\text {add }}\right)+\left(v_{1}+v_{2}\right) \quad(\bmod q),
\end{aligned}
$$

we obtain $f\left[c_{1}+c_{2}\right]_{q}=\Delta\left[m_{1}+m_{2}\right]_{t}+\left(v_{1}+v_{2}-r_{t}(q) r_{\text {add }}\right)(\bmod q)$ because $\Delta t \equiv-r_{t}(q)(\bmod q)$. This means that the size of the inherent noise $v_{\text {add }}$ of $c_{\text {add }}$ is bounded by

$$
\left\|v_{\text {add }}\right\|_{\infty} \leq\left\|v_{1}\right\|_{\infty}+\left\|v_{2}\right\|_{\infty}+r_{t}(q) .
$$

Up to the term $r_{t}(q)<t$, the inherent noise terms are added during homomorphic addition.

Homomorphic Multiplication. The homomorphic multiplication operation is divided into two parts. The first part describes a basic procedure to obtain an intermediate ciphertext that encrypts the product $\left[m_{1} m_{2}\right]_{t}$ modulo $t$ of two messages $m_{1}$ and $m_{2}$. However, the intermediate ciphertext can not be decrypted with Basic.Decrypt using the secret key $f$. The second part performs a procedure which allows a public transformation of this intermediate ciphertext to a ciphertext that can be decrypted with $f$. This latter procedure was introduced in [5] in the form of relinearization and was later expanded in [3] into a method called key switching, which transforms a ciphertext decryptable under one secret key to one decryptable under any other secret key. For our analysis, we assume that $\chi_{\mathrm{key}}$ and $\chi_{\mathrm{err}}$ are $B_{\mathrm{key}}{ }^{-}$and $B_{\mathrm{err}}$-bounded, respectively. Even if we work with unbounded Gaussian distributions, this is a valid assumption since elements drawn from either distribution have bounded norm for suitable bounds with high probability. The deduction of noise bounds mostly follows the basic multiplication section of [8], since ciphertexts and the decryption algorithm in YASHE have a very similar structure to those in [8]. 
First Step. Let $c_{1}, c_{2} \in R$ be ciphertexts that encrypt messages $m_{1}, m_{2} \in R$. In the first step of the homomorphic multiplication operation, we compute

$$
\tilde{c}_{\text {mult }}=\left[\left\lfloor\frac{t}{q} P_{w, q}\left(c_{1}\right) \otimes P_{w, q}\left(c_{2}\right)\right\rceil\right]_{q} .
$$

The following theorem shows that $\left\langle\tilde{c}_{\text {mult }}, D_{w, q}(f) \otimes D_{w, q}(f)\right\rangle=\Delta\left[m_{1} m_{2}\right]_{t}+\tilde{v}_{\text {mult }}$ $(\bmod q)$, and it provides a bound for the size of $\tilde{v}_{\text {mult }}$. Thus, $\tilde{c}_{\text {mult }}$ can be viewed as an encryption of $\left[m_{1} m_{2}\right]_{t}$ under $D_{w, q}(f) \otimes D_{w, q}(f)$ if the inherent noise term $\tilde{v}_{\text {mult }}$ is small enough.

Theorem 1 (Multiplication Noise). Let $c_{1}, c_{2} \in R$ be ciphertexts encrypting $m_{1}, m_{2} \in R$, decryptable with the secret key $f$. Let $v_{1}, v_{2} \in R$ be the inherent noise terms in $c_{1}, c_{2}$ and let $V>0$ such that $\left\|v_{i}\right\|_{\infty} \leq V<\Delta / 2$, $i \in\{1,2\}$. Let $\tilde{c}_{\text {mult }}$ be the intermediate ciphertext in YASHE.Mult, and let $\ell_{w, t B_{\mathrm{key}}}=\left\lfloor\log _{w}\left(t B_{\mathrm{key}}\right)\right\rfloor+2$. Then $\left\langle\tilde{c}_{\mathrm{mult}}, D_{w, q}(f) \otimes D_{w, q}(f)\right\rangle=\Delta\left[m_{1} m_{2}\right]_{t}+\tilde{v}_{\mathrm{mult}}$ $(\bmod q)$ where

$$
\left\|\tilde{v}_{\text {mult }}\right\|_{\infty}<\delta t\left(2+\delta \ell_{w, t B_{\mathrm{key}}} w\right) V+\frac{\delta t^{2}}{2}\left(3+\delta \ell_{w, t B_{\mathrm{key}}} w\right)+\frac{1}{8}\left(\delta \ell_{w, t B_{\mathrm{key}}} w\right)^{2}+\frac{1}{2} .
$$

Starting with two ciphertexts at a given inherent noise level, the first step of the multiplication increases the inherent noise level by a multiplicative factor of roughly $\delta^{2} t \ell_{w, t B_{\mathrm{key}}} w$ and an additive term of $\frac{\delta^{2}}{2} \ell_{w, t B_{\mathrm{key}}} w\left(t^{2}+\frac{1}{4} \ell_{w, t B_{\mathrm{key}}} w\right)$.

Key Switching. The second part in the homomorphic multiplication procedure is a key switching step, which transforms the ciphertext $\tilde{c}_{\text {mult }}$ into a ciphertext $c_{\text {mult }}$ that is decryptable under the original secret key $f$. We use the evaluation key

$$
\mathrm{evk}=\left[f^{-1} P_{w, q}\left(D_{w, q}(f) \otimes D_{w, q}(f)\right)+\boldsymbol{e}+h \cdot \boldsymbol{s}\right]_{q},
$$

output by YASHE. KeyGen where $\boldsymbol{e}, \boldsymbol{s} \leftarrow \chi_{\mathrm{err}}^{\ell_{w, q}^{3}}$ are vectors of polynomials sampled from the error distribution $\chi_{\mathrm{err}}$ and $[\cdot]_{q}$ is applied to each coefficient of the vector. Note that this key is a vector of quasi-encryptions of $f^{-1} P_{w, q}\left(D_{w, q}(f) \otimes D_{w, q}(f)\right)$ that depend on the secret key $f$, under its corresponding public key and that it is made public because it is needed for the homomorphic multiplication operation. Therefore, we need to make a circular security assumption, namely that the scheme is still secure even given that evk is publicly known (see Section 4.2). The following lemma deduces a bound on the noise caused by the key switching procedure and states an overall bound on the noise growth during a single homomorphic multiplication operation.

Lemma 3. Let notation be as in Theorem 1 and as above. In particular, let $\tilde{c}_{\text {mult }}$ be the intermediate ciphertext in YASHE. Mult with inherent noise term $\tilde{v}_{\text {mult }}$. Let evk be the evaluation key and $c_{\text {mult }}=$ YASHE.KeySwitch $\left(\tilde{c}_{\text {mult }}\right.$, evk $)$. Then $f c_{\text {mult }}=\Delta\left[m_{1} m_{2}\right]_{t}+v_{\text {mult }}(\bmod q)$, where

$$
\left\|v_{\text {mult }}\right\|_{\infty}<\left\|\tilde{v}_{\text {mult }}\right\|_{\infty}+\delta^{2} t \ell_{w, q}^{3} w B_{\text {err }} B_{\text {key }} .
$$


Theorem 1 and Lemma 3 give an overall upper bound on the noise growth during a homomorphic multiplication. This clearly dominates the noise growth for homomorphic addition. ${ }^{4}$

\subsection{Correctness}

This section discusses the correctness of YASHE and shows that it is a leveled homomorphic encryption scheme. We state correctness by giving an asymptotic bound on the number of multiplicative levels in an arithmetic circuit that can be correctly evaluated. For this, we concretely focus on a parameter setting such that the assumptions of the theorem by Stehle and Steinfeld (see Theorem 7 in Appendix A) hold. This means that the DSPR problem is hard in $R_{q}$. We therefore fix the following parameters: let $d$ be a power of $2, n=\varphi(d), \epsilon \in(0,1)$, $k \in(1 / 2,1)$ and let $q=2^{d^{\epsilon}}$ be a prime such that $\Phi_{d}(X)=X^{n}+1$ splits into $n$ irreducible factors modulo $q$. Let $\chi_{\text {key }}$ be a discrete Gaussian distribution on $R_{q}$ with deviation $\sigma_{\text {key }} \geq d \sqrt{\log (8 d q)} \cdot q^{k}$, and let $\chi_{\text {err }}$ be an asymptotically $\omega(\sqrt{d \log (d)})$-bounded Gaussian distribution on $R$ where $d$ tends to infinity. Finally, we fix $w=2$ and $t=2$, but note that similar results hold for general $w, t$ - this restriction is merely for the purpose of exposition.

Theorem 2 (Correctness of YASHE). For the parameter choices above, YASHE can evaluate any circuit of depth

$$
L=\mathcal{O}\left(\frac{(1-k) \log (q)}{\log (\log (q))+\log (d)}\right)
$$

\subsection{Security}

To prove security of YASHE, we need to assume that IND-CPA security can be maintained even when an adversary has access to elements of the evaluation key evk. Due to the way we construct evk it is not sufficient to simply replace $f$ by $L$ distinct secret keys $f_{i}$, as has been done in previous works - a specific assumption is still required. This is a form of key dependent message security, for the family of functions defining the evaluation key. Under this "circular security" assumption, the IND-CPA security of YASHE follows from the IND-CPA security of the scheme Basic described in Section 3 and the RLWE assumption.

Theorem 3 (Security of YASHE). The scheme YASHE is IND-CPA secure under the $\mathrm{RLWE}_{d, q, \chi_{\mathrm{err}}}$ assumption and the assumption that the scheme remains IND-CPA secure, even when an adversary has access to evk output by YASHE.KeyGen $\left(d, q, 2, \chi_{\mathrm{key}}, \chi_{\mathrm{err}}, 2\right)$.

\footnotetext{
${ }^{4}$ As noted in [2] the number of elements in $D_{w, q}(f) \otimes D_{w, q}(f)$ can be reduced from
} $\ell_{w, q}^{2}$ to $\left(\begin{array}{c}\ell_{w, q} \\ 2\end{array}\right)$ which correspondingly reduces the number of ring elements in evk. 
Proof. Since $\sigma_{\text {key }} \geq d \sqrt{\log (8 d q)} \cdot q^{k}$ for some $k>1 / 2+\nu$ with $\nu>0$, the conditions of Theorem 7 are satisfied. Hence the public key is indistinguishable from a uniform element of $R_{q}^{\times}$. It follows from [24] that the scheme Basic is IND-CPA secure under the RLWE $\mathrm{R}_{d, q, \chi_{\text {err }}}$ assumption in $R_{q}$. Under the circular security assumption outlined above, the IND-CPA security of YASHE follows.

For the proof of Theorem 3, we only need parameters that satisfy the assumptions in Theorem 7. For the parameters outlined at the beginning of this subsection, the RLWE assumption is believed to be hard based on standard worst-case lattice problems.

\subsection{From Leveled to Fully Homomorphic Encryption}

In [9], Gentry showed how a fully homomorphic scheme can be obtained from a leveled homomorphic scheme supporting computation of circuits of sufficient depth. If a scheme can evaluate its own decryption circuit and one additional multiplication, then that scheme can be converted to a fully homomorphic scheme. The only caveat is that we have to make an additional assumption: to execute the bootstrapping procedure, it is necessary to augment the public key with encryptions YASHE.Encrypt(pk, sk $[j])$ of the bits of the secret key, under its corresponding public key. Similarly to the assumption on the evaluation key, we need to make an additional assumption that including encryptions of bits of the secret key does not affect security.

To achieve a fully homomorphic scheme, we simply view the decryption circuit as a circuit computed on the bits of the secret key at a ciphertext $c$ we wish to refresh. The noise in the resulting fresh ciphertext will be of fixed size depending on the noise in the encryptions of the bits of the secret key. Lemma 6 and Theorem 8 in Appendix $\mathrm{H}$ show that YASHE can be bootstrapped to a fully homomorphic scheme.

\section{A More Practical Variant of the Scheme}

In this section, we propose a more practical variant YASHE' $^{\prime}$ of YASHE. The difference to YASHE lies in the homomorphic multiplication procedure. In YASHE', an intermediate ciphertext is simply a single polynomial while it is a vector of polynomials in YASHE. This results in an evaluation key that consists of only $\ell_{w, q}$ polynomials instead of $\ell_{w, q}^{3}$ for YASHE and thus in a simpler key switching procedure. We now state the scheme and discuss the noise growth during the simplified homomorphic multiplication operation YASHE' .Mult.

- YASHE'. ParamsGen $(\lambda)$ : Output $\left(d, q, t, \chi_{k e y}, \chi_{\text {err }}\right) \leftarrow \operatorname{BasicParamsGen}(\lambda)$.

- $\mathrm{YASHE}^{\prime} \cdot \operatorname{KeyGen}\left(d, q, t, \chi_{k e y}, \chi_{e r r}, w\right)$ : Compute

$$
h, f \leftarrow \text { Basic.KeyGen }\left(d, q, t, \chi_{\mathrm{key}}, \chi_{\mathrm{err}}\right) .
$$

Sample $\boldsymbol{e}, \boldsymbol{s} \leftarrow \chi_{\text {err }}^{\ell_{w, q}}$, compute $\gamma=\left[P_{w, q}(f)+\boldsymbol{e}+h \cdot s\right]_{q} \in R^{\ell_{w, q}}$. and output $(\mathrm{pk}, \mathrm{sk}, \mathrm{evk})=(h, f, \gamma)$. 
- YASHE ${ }^{\prime}$.Encrypt(pk, $\left.m\right)$ : Encrypt $m \in R$ as $c \leftarrow$ Basic.Encrypt(pk, $\left.m\right) \in R$.

- YASHE'.Decrypt(sk, c): Output the message $m \leftarrow$ Basic.Decrypt(sk, $c) \in R$.

- YASHE'.KeySwitch $\left(\tilde{c}_{\text {mult }}\right.$, evk): Output the ciphertext $\left[\left\langle D_{w, q}\left(\tilde{c}_{\text {mult }}\right), \text { evk }\right\rangle\right]_{q}$.

- YASHE' $\operatorname{Add}\left(c_{1}, c_{2}\right):$ Output $c_{a d d} \leftarrow$ YASHE. $\operatorname{Add}\left(c_{1}, c_{2}\right)=\left[c_{1}+c_{2}\right]_{q}$.

- YASHE'.Mult $\left(c_{1}, c_{2}\right.$, evk): Output the ciphertext

$$
c_{\text {mult }}=\mathrm{YASHE}^{\prime} \cdot \operatorname{KeyS} \text { witch }\left(\tilde{c}_{\text {mult }}, \text { evk }\right), \text { where } \tilde{c}_{\text {mult }}=\left[\left\lfloor\frac{t}{q} c_{1} c_{2}\right\rceil\right]_{q} .
$$

For two ciphertexts $c_{1}, c_{2} \in R$ that encrypt $m_{1}, m_{2} \in R$, the intermediate ciphertext $\tilde{c}_{\text {mult }}$ during homomorphic multiplication YASHE'. Mult satisfies $f^{2} \tilde{c}_{\text {mult }}=$ $\Delta\left[m_{1} m_{2}\right]_{t}+\tilde{v}_{\text {mult }}(\bmod q)$ as shown in the following theorem. This means that $\tilde{c}_{\text {mult }}$ is an encryption of $\left[m_{1} m_{2}\right]_{t}$ under $f^{2}$. The theorem also provides an upper bound on the inherent noise term in the intermediate ciphertext. We assume that the error distribution $\chi_{\mathrm{err}}$ is $B_{\mathrm{err}}$-bounded and that the key distribution $\chi_{\text {key }}$ is $B_{\text {key-bounded. }}$

Theorem 4 (Multiplication Noise). Let $c_{1}, c_{2} \in R$ be ciphertexts encrypting $m_{1}, m_{2} \in R$, which are decryptable with the secret key $f$. Let $v_{1}, v_{2} \in R$ be the inherent noise terms in $c_{1}, c_{2}$ and let $V>0$ such that $\left\|v_{i}\right\|_{\infty} \leq V<\Delta / 2$, $i \in\{1,2\}$. Let $\tilde{c}_{\text {mult }}$ be the intermediate ciphertext in $\mathrm{YASHE}^{\prime}$.Mult.

Then $f^{2} \tilde{c}_{\text {mult }}=\Delta\left[m_{1} m_{2}\right]_{t}+\tilde{v}_{\text {mult }}(\bmod q)$ where

$$
\left\|\tilde{v}_{\text {mult }}\right\|_{\infty}<\delta t\left(4+\delta t B_{\text {key }}\right) V+\delta^{2} t^{2} B_{\text {key }}\left(B_{\text {key }}+t\right) .
$$

Key Switching. The key switching algorithm now transforms such an intermediate encryption into a ciphertext that can be decrypted with $f$ itself. The evaluation key is evk $=\left[P_{w, q}(f)+\boldsymbol{e}+h \cdot s\right]_{q}$, where $\boldsymbol{e}, \boldsymbol{s} \leftarrow \chi_{\mathrm{err}}^{\ell_{w, q}}$ are vectors of polynomials sampled from the error distribution $\chi_{\mathrm{err}}$. Again, this key is a vector of quasi-encryptions of the secret key $f$ under its corresponding public key. It is required for the homomorphic multiplication operation and is therefore made public. This means, we need to make a circular security assumption as for YASHE, namely that the scheme is still secure even given that evk is publicly known. The following lemma gives a bound on the key switching noise.

Lemma 4. Let $\tilde{c}_{\text {mult }}$ be the intermediate ciphertext in $\mathrm{YASHE}^{\prime}$.Mult. Its inherent noise term is denoted by $\tilde{v}_{\text {mult }}$. Let $\boldsymbol{\gamma}$ be the evaluation key from above and $c_{\mathrm{mult}}=$ YASHE'. KeySwitch $\left(\tilde{c}_{\text {mult }}, \gamma\right)$. Then $f c_{\text {mult }}=\Delta\left[m_{1} m_{2}\right]_{t}+v_{\text {mult }}(\bmod q)$, where

$$
\left\|v_{\text {mult }}\right\|_{\infty}<\left\|\tilde{v}_{\text {mult }}\right\|_{\infty}+\delta^{2} t \ell_{w, q} w B_{\text {err }} B_{\text {key }} .
$$

\subsection{Correctness \& Security of YASHE'}

In the following theorem, we give an explicit bound for correctness of a homomorphic evaluation of an arithmetic circuit in $R / t R$ of multiplicative depth $L$ that is organized in a leveled tree structure of multiplications without any additions. At each level all ciphertexts are assumed to have inherent noise terms of 
roughly the same size. The bounds that we obtain might be too large and could be significantly reduced for computations that involve more additions and less multiplications as well as multiplications of ciphertexts with imbalanced inherent noise terms. In favor of simplicity, we restrict to the above setting.

Theorem 5 (Correctness of YASHE'). Let $\epsilon_{1}=4\left(\delta t B_{\mathrm{key}}\right)^{-1}$. The scheme YASHE' can correctly evaluate an arithmetic circuit consisting of L-levels of multiplications in $R / t R$ on ciphertexts with inherent noise of size at most $V$ that are arranged in a binary tree of $L$ levels of multiplications if

$2\left(1+\epsilon_{1}\right)^{L-1} \delta^{2 L} t^{2 L-1} B_{\mathrm{key}}^{L}\left(\left(1+\epsilon_{1}\right) t V+L\left(t B_{\mathrm{key}}+t^{2}+\ell_{w, q} w B_{\mathrm{err}}\right)\right)<\Delta-r_{t}(q)$.

Appendix K gives detailed bounds on the increase of the inherent noise terms in ciphertexts during homomorphic addition and multiplication. One can take these bounds to deduce overall bounds for the exact computation that is supposed to be carried out on encrypted data. The obtained bounds can then be used to deduce tailored parameters for the scheme to ensure correctness and security for that particular setting, possibly resulting in more efficient parameters for the specific computation.

The security of YASHE' is based on the RLWE assumption and a circular security assumption similar to the one for YASHE. The price we pay for a simpler homomorphic multiplication operation lies in an additional security assumption. Since YASHE' only works for a much narrower key distribution that does not satisfy the requirements for applying the Stehlé and Steinfeld result ([24, Thm. 4.1], see also Theorem 7 in Appendix A), security also relies on the Decisional Small Polynomial Ratio (DSPR) assumption, as stated in Section 2. In YASHE, this assumption could be avoided by making the scheme work with a key distribution as demanded by [24]. Following the same hybrid argument as in [15], one can prove that the scheme described in this section is secure under the DSPR assumption and the RLWE assumption (see [15, Section 3.3]). If $a, b$ are two elements sampled from a Gaussian with very small standard deviation or from a different distribution that yields polynomials with very small coefficients only, the ratio $h=a / b$ can clearly not be uniform because the number of elements for $a$ and $b$ is too small and produces only a small number of values for $h$ when compared to all elements in $R_{q}$. Still, a computationally bounded adversary might not be able to distinguish such a case from uniform randomly chosen $h$.

Theorem 6 (Security of YASHE'). Let $d$ be a positive integer, $q$ and $t<q$ be two moduli, $w$ be a fixed positive integer, and let $\chi_{\mathrm{key}}$ and $\chi_{\mathrm{err}}$ be distributions on $R$. The scheme $\mathrm{YASHE}^{\prime}$ is IND-CPA secure under the $\mathrm{RLWE}_{d, q, \chi_{\mathrm{err}}}$ assumption, the $\mathrm{DSPR}_{d, q, \chi_{\mathrm{key}}}$ assumption, and the assumption that the scheme remains IND-CPA secure even when the evaluation key evk which is output by $\mathrm{YASHE}^{\prime} . \operatorname{Key} \operatorname{Gen}\left(d, q, t, \chi_{\mathrm{key}}, \chi_{\mathrm{err}}\right)$ is known to the adversary.

Remark 1. The $\mathrm{DSPR}_{d, q, \chi_{\mathrm{key}}}$ assumption can be replaced by a weaker assumption $\operatorname{DSPR}_{d, q, \chi_{f}, \chi_{g}}$, where the elements $f$ and $g$ that are used for the public key $h=\left[t g f^{-1}\right]_{q}$ are sampled from distributions of different width with bounds $B_{f}$ 
and $B_{g}$, respectively. This new assumption can be made weaker than the original assumption since the element $g$ can be sampled from a much wider distribution than $f$. Introducing these two distributions means that the noise bound for the inherent noise in a fresh ciphertext is changed to $\delta t\left(B_{\text {err }}\left(B_{f}+B_{g}\right)+r_{t}(q) B_{f} / 2\right)$. The proofs of the noise bounds for YASHE' . Mult show that the bound $B_{g}$ only influences the constant $C_{2}$ in Lemma 9 . The contributions of $B_{g}$ in the noise bounds for $L$ levels of multiplications are merely a constant factor independent of $L$. Therefore, the scheme is still leveled homomorphic with the weaker assumption.

Remark 2. For $\mathrm{YASHE}^{\prime}$, since private keys are sampled with very small norm, the circular security assumption can be avoided in the usual way by providing a different public/private key pair $\left(h_{i}, f_{i}\right)$ for each level $i$ of multiplications for $0 \leq i \leq L$. The evaluation key has to be extended to $L$ vectors

$$
\gamma_{i}=\left[P_{w, q}\left(f_{i-1}^{2}\right)+e+h_{i} \cdot s\right]_{q},
$$

$1 \leq i \leq L$, such that the key switching step YASHE'. KeySwitch $\left(\tilde{c}_{\text {mult }}\right.$, evk $\left._{i}\right)$ transforms the intermediate ciphertext $\tilde{c}_{\text {mult }}$ decryptable under $f_{i-1}^{2}$ (obtained from two ciphertexts at level $i-1$ ) into one decryptable under $f_{i}$ at level $i$.

\subsection{Parameters}

In this section, we give suggestions for choosing concrete parameters which can be used as a guideline to instantiate practical schemes with varying complexity. There are multiple parameters one can adjust, so we restrict ourselves to a subset of choices which we think are most relevant. We consider two settings. In the first, we fix a specific size for the modulus $q$. This is interesting for instance when a fast modular multiplication implementation (in either hard- or software) is already available, and one prefers to use this to boost the scheme's performance. We fix different sizes for the modulus $q$ starting from 64 bits up to 1024 bits. The other setting focuses on special-purpose polynomial arithmetic. Here, we fix the degree $n=\varphi(d)$ to be a power of 2 between $2^{11}$ and $2^{16}$.

The parameters presented in Table 1 are obtained by following the security analysis of Lindner and Peikert [14] under the assumption that the results from [14] in the LWE setting carry over to the RLWE setting, and assuming that the assumptions in Section 5.1 hold. This analysis is similar to the ones from $[11,8,13]$ and we refer to [11] for a more complete discussion of assumptions made in deriving parameters. Note that recent results by Chen and Nguyen [6] are considered to be more accurate for estimating the security of specific parameters using the simulation of the BKZ 2.0 algorithm for assessing the runtime of lattice basis reduction. Selecting parameters for YASHE' with this method is ongoing work at the time of writing this paper. However, it is expected that the parameters presented in this paper which are obtained by using the LindnerPeikert method are more conservative than those obtained with the BKZ 2.0 simulation. 
Table 1. Parameters that guarantee security of $\lambda=80$ bits against the distinguishing attack with advantage $\epsilon=2^{-80}$. We fix $w=2^{32}$, the key distribution is assumed to be bounded by $B_{\text {key }}=1$, and we use $\sigma_{\text {err }}=8$ and $B_{\text {err }}=6 \sigma_{\mathrm{err}}$. Either for fixed sizes of $q$, we give the minimal degree $n_{\text {min }}$ (left part), or for fixed dimension $n$, we give the maximal size $\log \left(q_{\max }\right)$ (right part). For each pair $(q, n)$ according to the given sizes, and different values of $t$, correctness is guaranteed for at most $L_{\text {max }}$ multiplicative levels.

\begin{tabular}{|c|c|c|c|}
\hline$\lceil\log (q)\rceil$ & $n_{\min }$ & $t$ & $L_{\max }$ \\
\hline \multirow{3}{*}{64} & \multirow{3}{*}{1641} & 2 & 1 \\
\hline & & 256 & 0 \\
\hline & & 1024 & 0 \\
\hline \multirow{3}{*}{128} & \multirow{3}{*}{3329} & 2 & 3 \\
\hline & & 256 & 2 \\
\hline & & 1024 & 1 \\
\hline \multirow{3}{*}{192} & \multirow{3}{*}{5018} & 2 & 5 \\
\hline & & 256 & 3 \\
\hline & & 1024 & 3 \\
\hline \multirow{3}{*}{256} & \multirow{3}{*}{6707} & 2 & 7 \\
\hline & & 256 & 5 \\
\hline & & 1024 & 4 \\
\hline \multirow{3}{*}{512} & \multirow{3}{*}{13463} & 2 & 15 \\
\hline & & 256 & 10 \\
\hline & & 1024 & 9 \\
\hline \multirow{3}{*}{1024} & \multirow{3}{*}{26974} & 2 & 31 \\
\hline & & 256 & 21 \\
\hline & & 1024 & 19 \\
\hline
\end{tabular}

\begin{tabular}{|c|c|c|c|}
\hline$n$ & $\log \left(q_{\max }\right)$ & $t$ & $L_{\max }$ \\
\hline \multirow{3}{*}{$2^{11}$} & \multirow{3}{*}{79} & 2 & 1 \\
\hline & & 256 & 1 \\
\hline & & 1024 & 0 \\
\hline \multirow{3}{*}{$2^{12}$} & \multirow{3}{*}{157} & 2 & 4 \\
\hline & & 256 & 2 \\
\hline & & 1024 & 2 \\
\hline \multirow{3}{*}{$2^{13}$} & \multirow{3}{*}{312} & 2 & 9 \\
\hline & & 256 & 6 \\
\hline & & 1024 & 5 \\
\hline \multirow{3}{*}{$2^{14}$} & \multirow{3}{*}{622} & 2 & 19 \\
\hline & & 256 & 13 \\
\hline & & 1024 & 11 \\
\hline \multirow{3}{*}{$2^{15}$} & \multirow{3}{*}{1243} & 2 & 37 \\
\hline & & 256 & 25 \\
\hline & & 1024 & 23 \\
\hline \multirow{3}{*}{$2^{16}$} & \multirow{3}{*}{2485} & 2 & 71 \\
\hline & & 256 & 50 \\
\hline & & 1024 & 46 \\
\hline
\end{tabular}

Next, we discuss in more detail the parameter selection recommendations made in Table 1 . We use $B_{\text {key }}=1$, in other words we are assuming that even when the polynomials $f^{\prime}, g$ have coefficients in $\{-1,0,1\}$, the public key $h=$ $\left[t g f^{-1}\right]_{q}$ is indistinguishable from uniform. The standard deviation of the error distribution is fixed at $\sigma_{\mathrm{err}}=8$; this is consistent with [18]. The high probability bound on the size of the coefficients of errors drawn from Gaussian distributions is chosen as $6 \sigma_{\text {err }}$.

To distinguish with an advantage of $\epsilon$ in the RLWE problem, an adversary is required to find vectors of length at most $\alpha \cdot(q / \sigma)$ where $\alpha=\sqrt{\ln (1 / \epsilon) / \pi}$. In our specific parameter examples, we use $\epsilon=2^{-80}$, which results in $\alpha \approx 4.201$. We refer to [14] for a more complete description of a distinguishing attack and the precise lattices we are required to find short vectors in. Running SchnorrEuchner's BKZ [21], the best known lattice reduction algorithm in practice, and its successor BKZ 2.0 [6] for security parameter $\lambda$ (following [11] we use $\lambda=80)$ one expects to find vectors of length $2^{2 \sqrt{n \log _{2}(q) \log _{2}\left(\delta_{\mathrm{RHF}}\right)}}$ in time $T_{\mathrm{BKZ}}=$ $2^{\lambda}$ where $\delta_{\mathrm{RHF}}$ is the so-called root Hermite factor. This latter quantity is the overwhelming factor determining the quality of the basis which can be achieved 
in a given time and is computed as in [14] from

$$
\log _{2}\left(T_{\mathrm{BKZ}}\right)=1.8 / \log _{2}\left(\delta_{\mathrm{RHF}}\right)-110 .
$$

It is currently infeasible to achieve a target root Hermite factor $\delta_{\mathrm{RHF}}<1.005$ [6]. To guarantee security, we require that the shortest vector obtained through lattice reduction is longer than a vector which could give an adversary a nonnegligible advantage $\epsilon$ in the Ring-LWE distinguishing problem. This means that for security we thus require

$$
\alpha \cdot q / \sigma<2^{2 \sqrt{n \log _{2}(q) \log _{2}\left(\delta_{\mathrm{RHF}}\right)}} .
$$

For fixed parameters $\alpha$ and $\delta_{\mathrm{RHF}}$, this inequality provides bounds on the remaining parameters $q, \sigma_{\text {err }}$ and $n$. Fixing $\sigma_{\text {err }}$ too $\left(\sigma_{\text {err }}=8\right.$ here), we get a dependency between $q$ and $n$ that is expressed in the two settings discussed above as follows. When we fix $q$, we obtain a lower bound $n_{\min }$ for the dimension $n$ to guarantee security against the distinguishing attack. For the example values for the sizes of $q$ given in the first column of the left part of Table 1, we list this minimal degree in the second column. We used the worst case bound for a modulus $q$ of that size. Vice versa, first fixing the degree $n$ means that we get an upper bound $q_{\max }$ for $q$. We display the relation between $n$ and the size $\log \left(q_{\max }\right)$ in the first two columns of the right part of Table 1 .

For guaranteeing correctness, we use the noise bounds derived in the previous section. As mentioned in Section 2, when $d$ is a power of 2 and thus $\Phi_{d}(X)=$ $X^{n}+1$, the expansion factor is $\delta=n$. Then, by Lemma 1 and Lemma 9 we know that our scheme can correctly evaluate a depth $L$ circuit as long as

$$
\left(1+\epsilon_{1}\right)^{L-1} n^{2 L} t^{2 L-1} B_{\text {key }}^{L}\left(\left(1+\epsilon_{1}\right) t V+L\left(t\left(B_{\text {key }}+t\right)+\ell_{w, q} w B_{\text {err }}\right)\right)
$$

is less than $\left(\Delta-r_{t}(q)\right) / 2$, where $\epsilon_{1}=4\left(n t B_{\text {key }}\right)^{-1}$ and $V=n t B_{\text {key }}\left(2 B_{\text {err }}+\right.$ $\left.r_{t}(q) / 2\right)$ is the inherent noise of fresh ciphertexts by Lemma 2 . For each row in either the left or the right part of Table 1, we take the given values for $q$ and $n$ together with different values for $t$ and check what is the maximum number of levels $L_{\max }$ for which the correctness bound still holds. Note that in the left part, we take the minimal degree $n_{\min }$. This means that when choosing a power of 2 for the degree, the values for $L_{\max }$ might change. In the right part, we take the largest possible value for $q$ with the given maximal bit size.

It is important to ensure that the security bounds as well as the correctness bounds are both satisfied. Note that the authors of [8] failed to check their parameters presumably obtained from the correctness bound in the security bound, too, resulting in insecure parameters of $q=2^{1358}$ and $n=2^{10}$.

\subsection{Implementation}

Currently there are not many known implementation results for FHE schemes. Some of those which have been published demonstrate that the current stateof-the-art's performance is still rather unsatisfactory, see for example the implementations which are capable of computing AES homomorphically [11,7]. Other 
people have focused on implementing relatively simple schemes that require only a few levels of multiplications [13]. When using the ring $R=\mathbb{Z}[X] /\left(X^{4096}+1\right)$, $t=2^{10}$ and a 130-bit prime $q$, the authors of [13] present implementation results on an Intel Core 2 Duo running at $2.1 \mathrm{GHz}$. Encryption takes $756 \mathrm{~ms}$, addition of ciphertexts $4 \mathrm{~ms}$, multiplication of ciphertexts $1590 \mathrm{~ms}$ (this includes the degree reduction) and decryption $57 \mathrm{~ms}$.

We have implemented the YASHE' variant proposed in Section 5 in a Clibrary. All the arithmetic has been built from scratch and we do not depend on any external number theory library. Using almost the same parameters (we use a 127-bit prime $q$ ) with $w=2^{32}$ we obtained the following results on an Intel Core i7-3520M at $2893.484 \mathrm{MHz}$ with hyperthreading turned off and overclocking ("turbo boost") disabled. Encryption runs in 79.2 million cycles (27 $\mathrm{ms})$, addition of ciphertexts in 70 thousand cycles $(0.024 \mathrm{~ms})$, multiplication of ciphertexts (including the key-switching) in 90.7 million cycles $(31 \mathrm{~ms})$ and decryption in 14.1 million cycles $(5 \mathrm{~ms})$.

This performance increase by at least one order of magnitude (for the decryption) to two orders of magnitude (for the addition of ciphertexts) can be partially explained by the fact that we are running on a more recent processor and that we implemented the scheme directly in $\mathrm{C}$ (avoiding the overhead incurred by using a computer algebra system as in [13]). The remainder of the speed-up is due to our newly proposed scheme, in particular due to a simpler multiplication operation on ciphertexts that uses a more compact evaluation key consisting of only 4 elements. These performance numbers highlight the fact that HE is much more practical for schemes which do not require very deep circuits (like AES) but instead only need a few (around $2^{2}$ to $2^{5}$ ) multiplications.

\subsection{Truncating Ciphertext Words}

Brakerski [2, Section 4.2] first suggested for his scale-invariant LWE scheme to discard some least significant bits of the ciphertext. Based on this idea, we describe an optimization to our scheme which significantly reduces both the ciphertext length and the number of elements in the evaluation key. By aligning the number of bits we discard with a multiple of $w$ used in YASHE.KeySwitch, the number of elements required to switch keys is reduced per multiplication.

Define YASHE.Discard $\mathrm{w}_{\mathrm{w}}(c, i)$ as the function which takes as input a ciphertext and the number $0 \leq i<\ell_{w, q}$ of $w$-words to be truncated and outputs $c^{\prime}=\mathrm{YASHE} \operatorname{Discard}_{\mathrm{w}}(c, i)=\left\lfloor w^{-i} c\right\rfloor$. Then, $w^{i} c^{\prime}$ is equal to $c$ with the $i$ least significant $w$-words of $c$ being set to 0 . If $c f=\Delta m+v(\bmod q)$, then $w^{i} c^{\prime} f=$ $\Delta m+v^{\prime}(\bmod q)$ with $\left\|v^{\prime}\right\|_{\infty} \leq\|v\|_{\infty}+\frac{1}{2} \delta w^{i}\|f\|_{\infty}$. For a constant $B>0$ such that $2 B>\delta\|f\|_{\infty} / 2$, if we discard $\log _{w}(2 B)-\log _{w}\left(\delta\|f\|_{\infty}\right)$ words, we incur an additional noise term of size $B$, but the ciphertext can now be represented by $\log _{w}(q / B)+\log _{w}\left(\delta\|f\|_{\infty} / 2\right)$ words. This means that, with discarding, the length of ciphertexts does not depend on the absolute value of $q$ but only on the ratio of $q$ to the noise in the ciphertext. Perhaps more importantly, this means that when we consider $D_{w, q}(c)$ for a ciphertext $c$ with coefficients represented by roughly $\log _{w}(q / B)$ words, all the lowest $\log _{w}(B)$ words are now zero. If $c$ is a ciphertext 
decryptable under $f^{2}$, in the key switching step, we only need the top $\log _{w}(q / B)$ elements from the evaluation key to carry out the switch.

\subsection{Encoding input data via the CRT}

For our leveled homomorphic encryption scheme, we have given bounds on parameters and input data to ensure correctness and security. For applications such as outsourcing of storage and computation on private data to the cloud, it could be the case that the user requires a flexible system which allows for additional computation, more computation than was planned for when setting system parameters. We propose a way to extend the system to allow additional computation without resetting the parameters. For computations on integer values, the encoding of larger integers using the Chinese Remainder theorem allows for either greater precision of computation or larger integer inputs, using the same underlying field size and lattice dimension but at the cost of increasing the number of ciphertexts to be operated on.

Integer computations with results up to a bound $B$ are done by encoding each input as a collection of integers modulo coprime $t_{i}$ via the CRT. Computations are then carried out on the collection and correctly reflect the integer operations not involving any modular reductions, as long as the product of the $t_{i}$ is greater than $B$. Each integer in the collection is encrypted as a separate ciphertext with respect to its corresponding plain text modulus $t_{i}$ and those ciphertexts can be processed in parallel to return encrypted collections. After they are decrypted, the CRT is used to recover the output as an actual integer.

This approach is different than the ones introduced in [22] and [7], since in contrast to these schemes, we do not use the CRT to pack information into different plain text slots of a single ciphertext. Instead, we simply encrypt each part of the CRT encoding in a separate ciphertext with respect to its plain text modulus $t_{i}$. This introduces a different way of flexibility. Ciphertexts now consist of several ring elements, but can be processed in parallel. For example, this allows to work on integers of double bit length by keeping the same parameters, only extending to two ciphertexts with different values for $t_{0}$ and $t_{1}$.

\section{Conclusions}

We have proposed a new fully homomorphic encryption scheme based on the scheme by Stehlé and Steinfeld which removes the non-standard decisional small polynomial ratio assumption needed in the homomorphic encryption scheme by López-Alt, Tromer and Vaikuntanathan. Hence, the security is solely based on standard lattice assumptions and a circular security assumption. Our new scheme avoids modulus switching and keeps the size of ciphertexts to a single ring element. Furthermore, we have presented a more practical variant of our scheme which does need the decisional small polynomial ratio assumption. For this latter scheme we presented parameters and implementation results. 
Acknowledgments. The authors thank Adriana López-Alt for many useful suggestions and discussions, in particular for pointing out the possibility of a weaker assumption in Remark 1, Tancrède Lepoint for very useful comments and for noticing an error in an earlier version of Table 1, Nigel P. Smart for helpful advice and the anonymous reviewers for their constructive feedback.

\section{References}

1. B. Applebaum, D. Cash, C. Peikert, and A. Sahai. Fast cryptographic primitives and circular-secure encryption based on hard learning problems. In Advances in Cryptology - CRYPTO 2009, 29th Annual International Cryptology Conference, volume 5677 of Lecture Notes in Computer Science, pages 595-618. Springer, 2009.

2. Z. Brakerski. Fully homomorphic encryption without modulus switching from classical GapSVP. In Advances in Cryptology - Crypto 2012, volume 7417 of Lecture Notes in Computer Science, pages 868-886. Springer, 2012.

3. Z. Brakerski, C. Gentry, and V. Vaikuntanathan. Fully homomorphic encryption without bootstrapping. In ITCS, pages 309-325, 2012.

4. Z. Brakerski and V. Vaikuntanathan. Efficient fully homomorphic encryption from (standard) LWE. In FOCS, pages 97-106, 2011.

5. Z. Brakerski and V. Vaikuntanathan. Fully homomorphic encryption from ringLWE and security for key dependent messages. In CRYPTO, pages 505-524, 2011.

6. Y. Chen and P. Q. Nguyen. BKZ 2.0: Better lattice security estimates. In ASIACRYPT, volume 7073 of Lecture Notes in Computer Science, pages 1-20. Springer, 2011.

7. J. H. Cheon, J.-S. Coron, J. Kim, M. S. Lee, T. Lepoint, M. Tibouchi, and A. Yun. Batch fully homomorphic encryption over the integers. In T. Johansson and P. Q. Nguyen, editors, EUROCRYPT, volume 7881 of Lecture Notes in Computer Science, pages 315-335. Springer, 2013.

8. J. Fan and F. Vercauteren. Somewhat practical fully homomorphic encryption. Cryptology ePrint Archive, Report 2012/144, 2012. http://eprint.iacr.org/.

9. C. Gentry. Fully homomorphic encryption using ideal lattices. In STOC, pages 169-178, 2009.

10. C. Gentry and S. Halevi. Implementing gentry's fully-homomorphic encryption scheme. In Advances in Cryptology - EUROCRYPT 2011 - 30th Annual International Conference on the Theory and Applications of Cryptographic Techniques, volume 6632 of Lecture Notes in Computer Science, page 129. Springer, 2011.

11. C. Gentry, S. Halevi, and N. P. Smart. Homomorphic evaluation of the AES circuit. In Advances in Cryptology - Crypto 2012, volume 7417 of Lecture Notes in Computer Science, pages 850-867. Springer, 2012.

12. J. Hoffstein, J. Pipher, and J. H. Silverman. NTRU: A ring-based public key cryptosystem. In J. Buhler, editor, ANTS, volume 1423 of Lecture Notes in Computer Science, pages 267-288. Springer, 1998.

13. K. Lauter, M. Naehrig, and V. Vaikuntanathan. Can homomorphic encryption be practical? In C. Cachin and T. Ristenpart, editors, CCSW, pages 113-124. ACM, 2011.

14. R. Lindner and C. Peikert. Better key sizes (and attacks) for LWE-based encryption. In Proceedings of the 11th international conference on Topics in cryptology: CT-RSA 2011, CT-RSA'11, pages 319-339, Berlin, Heidelberg, 2011. SpringerVerlag. 
15. A. López-Alt, E. Tromer, and V. Vaikuntanathan. On-the-fly multiparty computation on the cloud via multikey fully homomorphic encryption. In $S T O C$, pages 1219-1234, 2012.

16. V. Lyubashevsky, C. Peikert, and O. Regev. On ideal lattices and learning with errors over rings. In Advances in Cryptology - EUROCRYPT 2010, 29th Annual International Conference on the Theory and Applications of Cryptographic Techniques, volume 6110 of Lecture Notes in Computer Science, pages 1-23. Springer, 2010.

17. D. Micciancio and O. Regev. Worst-case to average-case reductions based on Gaussian measures. In SIAM J. on Computing, pages 372-381. IEEE Computer Society, 2004.

18. D. Micciancio and O. Regev. Lattice-based cryptography. In D. J. Bernstein, J. Buchmann, and E. Dahmen, editors, Post-Quantum Cryptography, pages 147191. Springer Berlin Heidelberg, 2009.

19. D. Naccache, J.-S. Coron, A. Mandal, and M. Tibouchi. Fully homomorphic encryption over the integers with shorter public keys. In Advances in Cryptology CRYPTO 2011 - 31st Annual Cryptology Conference, volume 6841 of Lecture Notes in Computer Science, page 483. Springer, 2011.

20. R. L. Rivest, L. Adleman, and M. L. Dertouzos. On data banks and privacy homomorphisms. In Foundations of secure computation, volume 4, pages 169-180. New-York: Academic Press, 1978.

21. C.-P. Schnorr. A hierarchy of polynomial time lattice basis reduction algorithms. Theor. Comput. Sci., 53:201-224, 1987.

22. N. Smart and F. Vercauteren. Fully homomorphic simd operations. Cryptology ePrint Archive, Report 2011/133, 2011. http://eprint.iacr.org/.

23. N. P. Smart and F. Vercauteren. Fully homomorphic encryption with relatively small key and ciphertext sizes. In Public Key Cryptography - PKC 2010, 13th International Conference on Practice and Theory in Public Key Cryptography, Paris, France, May 26-28, 2010. Proceedings, volume 6056 of Lecture Notes in Computer Science, pages 420-443. Springer, 2010.

24. D. Stehle and R. Steinfeld. Making NTRU as secure as worst-case problems over ideal lattices. In Advances in Cryptology - EUROCRYPT 2011 - 30th Annual International Conference on the Theory and Applications of Cryptographic Techniques, volume 6632 of Lecture Notes in Computer Science, page 27. Springer, 2011.

25. D. Stehle and R. Steinfeld. Making NTRU as secure as worst-case problems over ideal lattices. Cryptology ePrint Archive, Report 2013/004, 2012.

26. M. van Dijk, C. Gentry, S. Halevi, and V. Vaikuntanathan. Fully homomorphic encryption over the integers. In Advances in Cryptology - EUROCRYPT 2010, 29th Annual International Conference on the Theory and Applications of Cryptographic Techniques, volume 6110 of Lecture Notes in Computer Science, pages 24-43. Springer, 2010.

\section{A The Stehlé-Steinfeld theorem}

Let $d \geq 8$ be a power of 2 such that $\Phi_{d}(X)=X^{d / 2}+1$ splits into $k_{q}$ irreducible factors modulo a prime $q \geq 5$. Define $R=\mathbb{Z}[X] /\left(\Phi_{d}(X)\right)$, and $R_{q}=R / q R$. Let $U\left(R_{q}^{\times}\right)$be the uniform distribution on $R_{q}^{\times}$, the set of invertible elements in $R_{q}$. Let $\chi=D_{\mathbb{Z}^{d / 2}, \sigma}^{\times}$be the spherical discrete Gaussian distribution on $R_{q}$, restricted to $R_{q}^{\times}$, and for $z \in R_{q}$, let $\chi_{z}=\chi+z$ be the Gaussian shifted by $z$. 
Theorem 7 (Stehlé and Steinfeld [25]). Let $0<\epsilon<1 / 3, t \in R_{q}^{\times}$, $\chi$ and $\sigma$ as above, $y_{i} \in R_{q}$ and $z_{i}=-y_{i} t^{-1}(\bmod q)$ for $i \in\{1,2\}$. Then the statistical distance $D$ between distributions $\frac{y_{1}+t \cdot \chi_{z_{1}}}{y_{2}+t \cdot \chi_{z_{2}}}(\bmod q)$ and $U\left(R_{q}^{\times}\right)$is bounded by

$$
D \leq \begin{cases}2^{10 d} \cdot q^{-\frac{\left\lfloor\epsilon k_{q}\right\rfloor}{k_{q}} \cdot d} & \text { if } \sigma \geq d \cdot \sqrt{\log (8 d q)} \cdot q^{\frac{1}{2}+\epsilon}, \\ 2^{10 d} \cdot q^{-\epsilon d} & \text { if } \sigma \geq \sqrt{d \log (8 d q)} \cdot q^{\frac{1+k_{q} \epsilon}{2}} \text { and } q \geq d^{\frac{k_{q}}{1-2 k_{q} \epsilon}} .\end{cases}
$$

\section{B Proof of Lemma 1}

Since $f c=\Delta[m]_{t}+v(\bmod q)$, we can write $[f c]_{q}=\Delta[m]_{t}+v+q a$ for some $a \in R$ and compute

$$
t / q \cdot[f c]_{q}=(t / q) \Delta[m]_{t}+v \cdot t / q+t a .
$$

Using $q=\Delta t+r_{t}(q)$ and setting $\epsilon=r_{t}(q) / t$, this yields $t / q \cdot[f c]_{q}=[m]_{t}+$ $t / q\left(v-(q / t-\Delta)[m]_{t}\right)+t a=[m]_{t}+t / q\left(v-\epsilon[m]_{t}\right)+t a$. The assumption on the norm of $v$ implies $\left\|v-\epsilon[m]_{t}\right\|_{\infty}<\left(\Delta-r_{t}(q)\right) / 2+r_{t}(q) / 2=\Delta / 2 \leq q /(2 t)$, i.e. $\left\|t / q\left(v-\epsilon[m]_{t}\right)\right\|_{\infty}<1 / 2$. Therefore, rounding of $[f c]_{q}$ gives $[m]_{t}+t a$ and thus $c$ decrypts correctly.

\section{Proof of Lemma 2}

We have $c=\Delta[m]_{t}+e+h s$ for $e, s \leftarrow \chi_{\mathrm{err}}$. Using $\Delta t=-r_{t}(q)(\bmod q)$, it follows that

$$
f c=\Delta f[m]_{t}+f e+t g s=\Delta[m]_{t}-r_{t}(q) f^{\prime}[m]_{t}+f e+t g s \quad(\bmod q) .
$$

Define $v=f e+t g s-r_{t}(q) f^{\prime}[m]_{t}$. Since $f^{\prime}, g \leftarrow \chi_{\text {key }}$ and $s, e \leftarrow \chi_{\mathrm{err}}$, we obtain $\|f e\|_{\infty},\|t g s\|_{\infty}<\delta t B_{\text {key }} B_{\text {err }}$ and $\left\|f^{\prime}[m]_{t}\right\|_{\infty}<\delta B_{\text {key }} t / 2$. Altogether, $\|v\|_{\infty}<2 \delta t B_{\text {key }} B_{\text {err }}+r_{t}(q) \delta t B_{\text {key }} / 2$.

\section{A Useful Tool}

Lemma 5. Let $a_{1}, a_{2}, m_{1}, m_{2}, v_{1}, v_{2} \in R$ such that $\left\|v_{i}\right\|_{\infty}<\Delta / 2$, and $a_{i}=$ $\Delta\left[m_{i}\right]_{t}+v_{i}+q r_{i}$ for polynomials $r_{i} \in R$. Let $\left[m_{1}\right]_{t}\left[m_{2}\right]_{t}=\left[m_{1} m_{2}\right]_{t}+t r_{m}$ and $v_{1} v_{2}=\left[v_{1} v_{2}\right]_{\Delta}+\Delta r_{v}$ where $r_{m}, r_{v} \in R$. Then $\left\|r_{m}\right\|_{\infty}<\frac{1}{2} \delta t,\left\|r_{v}\right\|_{\infty} \leq$ $\frac{1}{2} \delta \min _{i}\left\|v_{i}\right\|_{\infty}$ and

$$
\begin{aligned}
\frac{t}{q} a_{1} a_{2}= & \Delta\left[m_{1} m_{2}\right]_{t}+\left[m_{1}\right]_{t} v_{2}+\left[m_{2}\right]_{t} v_{1}+t\left(v_{1} r_{2}+r_{1} v_{2}\right) \\
& -r_{t}(q)\left(\left[m_{1}\right]_{t} r_{2}+\left[m_{2}\right]_{t} r_{1}+r_{m}\right)+r_{v}+r_{r}+q s
\end{aligned}
$$

where $s \in R$ and $r_{r}=\frac{t}{q}\left[v_{1} v_{2}\right]_{\Delta}-\frac{r_{t}(q)}{q}\left(\Delta\left[m_{1}\right]_{t}\left[m_{2}\right]_{t}+\left[m_{1}\right]_{t} v_{2}+\left[m_{2}\right]_{t} v_{1}+r_{v}\right) \in \frac{1}{q} R$. Note that all terms on the right hand side of the equation are elements of $R$, except possibly $r_{r}$, which is bounded by $\left\|r_{r}\right\|_{\infty}<\frac{1}{2}+r_{t}(q) \delta t$. 
Proof. The bounds on $r_{m}$ and $r_{v}$ can be derived as follows. First, we have $\left\|r_{m}\right\|_{\infty}=\frac{1}{t}\left\|\left[m_{1}\right]_{t}\left[m_{2}\right]_{t}-\left[m_{1} m_{2}\right]_{t}\right\|_{\infty} \leq \frac{1}{t}\left(\delta \frac{t^{2}}{4}+\frac{t}{2}\right)=\delta \frac{t}{4}+\frac{1}{2}<\frac{1}{2} \delta t$. Similarly, $\left\|r_{v}\right\|_{\infty} \leq \frac{1}{\Delta} \delta\left\|v_{1}\right\|_{\infty}\left\|v_{2}\right\|_{\infty}+\frac{1}{2}<\frac{1}{2} \delta \min _{i}\left\|v_{i}\right\|_{\infty}+\frac{1}{2}$, i.e. $2\left\|r_{v}\right\|_{\infty}<\delta \min _{i}\left\|v_{i}\right\|_{\infty}+$ 1. Since $r_{v} \in R$, we get $2\left\|r_{v}\right\|_{\infty} \leq \delta \min _{i}\left\|v_{i}\right\|_{\infty}$. Multiplying out and making use of the equality $q-r_{t}(q)=\Delta t$, we obtain

$$
\begin{aligned}
\frac{t}{q} \cdot a_{1} a_{2}= & \frac{\Delta t}{q} \Delta\left[m_{1}\right]_{t}\left[m_{2}\right]_{t}+\frac{\Delta t}{q}\left(\left[m_{1}\right]_{t} v_{2}+\left[m_{2}\right]_{t} v_{1}\right)+t\left(v_{1} r_{2}+r_{1} v_{2}\right)+\frac{t}{q} v_{1} v_{2} \\
& +\Delta t\left(\left[m_{1}\right]_{t} r_{2}+r_{1}\left[m_{2}\right]_{t}\right)+q t r_{1} r_{2} \\
= & \Delta\left[m_{1}\right]_{t}\left[m_{2}\right]_{t}-\frac{r_{t}(q)}{q} \Delta\left[m_{1}\right]_{t}\left[m_{2}\right]_{t}+\left[m_{1}\right]_{t} v_{2}+\left[m_{2}\right]_{t} v_{1} \\
& -\frac{r_{t}(q)}{q}\left(\left[m_{1}\right]_{t} v_{2}+\left[m_{2}\right]_{t} v_{1}\right)+t\left(v_{1} r_{2}+v_{2} r_{1}\right)+\frac{t}{q} v_{1} v_{2} \\
& +q\left(\left[m_{1}\right]_{t} r_{2}+r_{1}\left[m_{2}\right]_{t}\right)-r_{t}(q)\left(\left[m_{1}\right]_{t} r_{2}+\left[m_{2}\right]_{t} r_{1}\right)+q t r_{1} r_{2} \\
= & \Delta\left[m_{1}\right]_{t}\left[m_{2}\right]_{t}+\left[m_{1}\right]_{t} v_{2}+\left[m_{2}\right]_{t} v_{1}+t\left(v_{1} r_{2}+r_{1} v_{2}\right) \\
& -r_{t}(q)\left(\left[m_{1}\right]_{t} r_{2}+\left[m_{2}\right]_{t} r_{1}\right)-\frac{r_{t}(q)}{q}\left(\Delta\left[m_{1}\right]_{t}\left[m_{2}\right]_{t}+\left[m_{1}\right]_{t} v_{2}+\left[m_{2}\right]_{t} v_{1}\right) \\
& +\frac{t}{q} v_{1} v_{2}+q\left(t r_{1} r_{2}+\left[m_{1}\right]_{t} r_{2}+\left[m_{2}\right]_{t} r_{1}\right) \\
= & \Delta\left[m_{1} m_{2}\right]_{t}+\left[m_{1}\right]_{t} v_{2}+\left[m_{2}\right]_{t} v_{1}+t\left(v_{1} r_{2}+r_{1} v_{2}\right) \\
& -r_{t}(q)\left(\left[m_{1}\right]_{t} r_{2}+\left[m_{2}\right]_{t} r_{1}+r_{m}\right)+r_{v} \\
& -\frac{r_{t}(q)}{q}\left(\Delta\left[m_{1}\right]_{t}\left[m_{2}\right]_{t}+\left[m_{1}\right]_{t} v_{2}+\left[m_{2}\right]_{t} v_{1}+r_{v}\right)+\frac{t}{q}\left[v_{1} v_{2}\right]_{\Delta} \\
& +q\left(t r_{1} r_{2}+\left[m_{1}\right]_{t} r_{2}+\left[m_{2}\right]_{t} r_{1}+r_{m}\right) .
\end{aligned}
$$

With $s=t r_{1} r_{2}+\left[m_{1}\right]_{t} r_{2}+\left[m_{2}\right]_{t} r_{1}+r_{m} \in R$, we obtain the equation for $\frac{t}{q} f^{2} c_{1} c_{2}$ with $r_{r}$ as given above. The bound for $\left\|r_{r}\right\|_{\infty}$ follows from

$$
\begin{aligned}
\left\|r_{r}\right\|_{\infty} & =\frac{1}{q}\left\|t\left[v_{1} v_{2}\right]_{\Delta}-r_{t}(q)\left(\Delta\left[m_{1}\right]_{t}\left[m_{2}\right]_{t}+\left[m_{1}\right]_{t} v_{2}+\left[m_{2}\right]_{t} v_{1}+r_{v}\right)\right\|_{\infty} \\
& \leq \frac{1}{q}\left(\frac{\Delta t}{2}+r_{t}(q)\left(\delta \frac{\Delta t^{2}}{4}+\delta \frac{t}{2}\left(\left\|v_{1}\right\|_{\infty}+\left\|v_{2}\right\|_{\infty}\right)+\left\|r_{v}\right\|_{\infty}\right)\right) \\
& <\frac{1}{2}+r_{t}(q) \delta\left(\frac{t}{4}+\frac{1}{2}+\frac{1}{4 t}\right)<\frac{1}{2}+r_{t}(q) \delta t,
\end{aligned}
$$

where we have used the bound on $\left\|r_{v}\right\|_{\infty}$ and the fact that $\left\|v_{i}\right\|_{\infty}<\Delta / 2$.

\section{E Proof of Theorem 1}

To analyse how large $\tilde{v}_{\text {mult }}$ is, let $v_{1}, v_{2} \in R$ be the inherent noise terms in $c_{1}, c_{2}$. Using $f c_{i}=\Delta\left[m_{i}\right]_{t}+v_{i}(\bmod q)$ and $\left\langle P_{w, q}\left(c_{i}\right), D_{w, q}(f)\right\rangle=f c_{i}(\bmod q)$ for $i \in\{1,2\}$, this means we can write

$$
\left\langle P_{w, q}\left(c_{i}\right), D_{w, q}(f)\right\rangle=\Delta\left[m_{i}\right]_{t}+v_{i}+q \cdot r_{i}
$$


for polynomials $r_{i} \in R$. We assume that the assumptions in Lemma 1 are satisfied and that the $v_{i}$ are chosen such that $\left\|v_{i}\right\|_{\infty}<\left(\Delta-r_{t}(q)\right) / 2$. In particular, $c_{1}, c_{2}$ are decryptable. Since the coefficients of $P_{w, q}\left(c_{i}\right)$ are bounded by $q / 2$ in absolute value, those of $D_{w, q}(f)$ by $w / 2$, and $D_{w, q}(f)$ has at most $\ell_{w, t B_{\mathrm{key}}}=$ $\left\lfloor\log _{w}\left(t B_{\text {key }}\right)\right\rfloor+2$ non-zero entries, the polynomials $r_{i}$ can be bounded by

$$
\left\|r_{i}\right\|_{\infty} \leq \frac{1}{q}\left(\delta \ell_{w, t B_{\mathrm{key}}} \frac{q}{2} \cdot \frac{w}{2}+\Delta \frac{t}{2}+\left\|v_{i}\right\|_{\infty}\right) \leq \frac{1}{4} \delta \ell_{w, t B_{\mathrm{key}}} w+1<\frac{1}{2} \delta \ell_{w, t B_{\mathrm{key}}} w .
$$

Note that we have used $\Delta \leq q / t,\left\|v_{i}\right\|_{\infty}<\Delta / 2$ and the definition of $\delta$. Multiplying the scalar products $\left\langle P_{w, q}\left(c_{1}\right), D_{w, q}(f)\right\rangle$ and $\left\langle P_{w, q}\left(c_{2}\right), D_{w, q}(f)\right\rangle$, using $\left\langle P_{w, q}\left(c_{1}\right) \otimes P_{w, q}\left(c_{2}\right), D_{w, q}(f) \otimes D_{w, q}(f)\right\rangle=\left\langle P_{w, q}\left(c_{1}\right), D_{w, q}(f)\right\rangle\left\langle P_{w, q}\left(c_{2}\right), D_{w, q}(f)\right\rangle$ and substituting Equation (2) yields

$$
\begin{aligned}
\left\langle P_{w, q}\left(c_{1}\right) \otimes P_{w, q}\left(c_{2}\right), D_{w, q}(f) \otimes D_{w, q}(f)\right\rangle= & \Delta^{2}\left[m_{1}\right]_{t}\left[m_{2}\right]_{t}+\Delta\left(\left[m_{1}\right]_{t} v_{2}+\left[m_{2}\right]_{t} v_{1}\right) \\
& +q\left(v_{1} r_{2}+r_{1} v_{2}\right)+v_{1} v_{2} \\
& +q \Delta\left(\left[m_{1}\right]_{t} r_{2}+r_{1}\left[m_{2}\right]_{t}\right)+q^{2} r_{1} r_{2}
\end{aligned}
$$

Next, we incorporate $\left[m_{1} m_{2}\right]_{t}$ into the above expression and scale by $t / q$. As observed in [8], simply scaling by $\Delta$ would give an additional error term caused by rounding of $q^{2} r_{1} r_{2}$. To make things more clear we expand the exposition of [8] for our scheme in Lemma 5, which is stated and proved in Appendix D. We obtain

$$
\begin{aligned}
\frac{t}{q}\left\langle P_{w, q}\left(c_{1}\right) \otimes P_{w, q}\left(c_{2}\right), D_{w, q}(f) \otimes D_{w, q}(f)\right\rangle= & \Delta\left[m_{1} m_{2}\right]_{t}+\left[m_{1}\right]_{t} v_{2} \\
& +\left[m_{2}\right]_{t} v_{1}+t\left(v_{1} r_{2}+r_{1} v_{2}\right) \\
& -r_{t}(q)\left(\left[m_{1}\right]_{t} r_{2}+\left[m_{2}\right]_{t} r_{1}+r_{m}\right) \\
& +r_{v}+r_{r}+q s
\end{aligned}
$$

where $r_{m}, r_{v}$, and $r_{r}$ are as in Lemma 5 and we have $\left\|r_{m}\right\|_{\infty}<\frac{1}{2} \delta t,\left\|r_{v}\right\|_{\infty} \leq$ $\frac{1}{2} \delta \min _{i}\left\|v_{i}\right\|_{\infty}$, and $\left\|r_{r}\right\|_{\infty}<\frac{1}{2}+r_{t}(q) \delta t$.

To bound the size of the inherent noise term in $\tilde{c}_{\text {mult }}$, we need to consider $\left\langle\tilde{c}_{\text {mult }}, D_{w, q}(f) \otimes D_{w, q}(f)\right\rangle$. Define

$$
\begin{aligned}
& r_{a}=\frac{t}{q}\left\langle P_{w, q}\left(c_{1}\right) \otimes P_{w, q}\left(c_{2}\right), D_{w, q}(f) \otimes D_{w, q}(f)\right\rangle-\left\langle\tilde{c}_{\text {mult }}, D_{w, q}(f) \otimes D_{w, q}(f)\right\rangle \\
&=\left\langle\left(\frac{t}{q} P_{w, q}\left(c_{1}\right) \otimes P_{w, q}\left(c_{2}\right)\right)-\left\lfloor\frac{t}{q} \cdot P_{w, q}\left(c_{1}\right) \otimes P_{w, q}\left(c_{2}\right)\right\rceil\right. \\
&\left.D_{w, q}(f) \otimes D_{w, q}(f)\right\rangle
\end{aligned}
$$

The coefficients of all polynomials in the vector in the left argument of the scalar product are bounded in absolute value by $1 / 2$, while those in the vector in the right argument are products of polynomials with coefficients bounded by $w / 2$. 
Both vectors have length $\ell_{w, q}^{2}$, but at most $\ell_{w, t B_{\mathrm{key}}}^{2}$ entries of $D_{w, q}(f) \otimes D_{w, q}(f)$ are non-zero, which means we get a bound on $r_{a}$ as

$$
\left\|r_{a}\right\|_{\infty} \leq \ell_{w, t B_{\mathrm{key}}}^{2} \delta \cdot \frac{1}{2} \cdot \delta\left(\frac{w}{2}\right)^{2}=\frac{1}{8}\left(\delta \ell_{w, t B_{\mathrm{key}}} w\right)^{2} .
$$

We are now in a position to bound the inherent noise term in the intermediate ciphertext $\tilde{c}_{\text {mult }}$ after the first part of the homomorphic multiplication procedure. Again, this is very similar to [8].

From Equations (4) and (5) we have $\left\langle\tilde{c}_{\text {mult }}, D_{w, q}(f) \otimes D_{w, q}(f)\right\rangle \equiv \frac{t}{q}\left\langle P_{w, q}\left(c_{1}\right) \otimes\right.$ $\left.P_{w, q}\left(c_{2}\right), D_{w, q}(f) \otimes D_{w, q}(f)\right\rangle-r_{a} \equiv \Delta\left[m_{1} m_{2}\right]_{t}+\tilde{v}_{\text {mult }}(\bmod q)$, where we define $\tilde{v}_{\text {mult }}=\left[m_{1}\right]_{t} v_{2}+\left[m_{2}\right]_{t} v_{1}+t\left(v_{1} r_{2}+r_{1} v_{2}\right)-r_{t}(q)\left(\left[m_{1}\right]_{t} r_{2}+\left[m_{2}\right]_{t} r_{1}+r_{m}\right)+r_{v}+r_{r}-r_{a}$.

It follows

$$
\begin{aligned}
\left\|\tilde{v}_{\text {mult }}\right\|_{\infty} \leq & \left\|\left[m_{1}\right]_{t} v_{2}+\left[m_{2}\right]_{t} v_{1}\right\|_{\infty}+t\left\|v_{1} r_{2}+r_{1} v_{2}\right\|_{\infty} \\
& +r_{t}(q)\left(\left\|\left[m_{1}\right]_{t} r_{2}+\left[m_{2}\right]_{t} r_{1}\right\|_{\infty}+\left\|r_{m}\right\|_{\infty}\right) \\
& +\left\|r_{v}\right\|_{\infty}+\left\|r_{r}\right\|_{\infty}+\left\|r_{a}\right\|_{\infty} .
\end{aligned}
$$

We bound the summands separately and add together to obtain the overall bound. We have $\left\|\left[m_{1}\right]_{t} v_{2}+\left[m_{2}\right]_{t} v_{1}\right\|_{\infty} \leq \delta \frac{t}{2}\left(\left\|v_{1}\right\|_{\infty}+\left\|v_{2}\right\|_{\infty}\right)$, since the coefficients of the $\left[m_{i}\right]_{t}$ are of absolute value at most $t / 2$. Similarly, the bounds on $\left\|r_{i}\right\|_{\infty}$ lead to $\left\|v_{1} r_{2}+r_{1} v_{2}\right\|_{\infty} \leq \delta \cdot \frac{1}{2} \delta \ell_{w, t B_{\mathrm{key}}} w\left(\left\|v_{1}\right\|_{\infty}+\left\|v_{2}\right\|_{\infty}\right)$, and $\|\left[m_{1}\right]_{t} r_{2}+$ $\left[m_{2}\right]_{t} r_{1} \|_{\infty} \leq \delta t \frac{1}{2} \delta \ell_{w, t B_{\mathrm{key}}} w$. We have already obtained bounds for $\left\|r_{m}\right\|_{\infty},\left\|r_{v}\right\|_{\infty}$, $\left\|r_{r}\right\|_{\infty}$, and $\left\|r_{a}\right\|_{\infty}$ above. We summarize and get

$$
\begin{aligned}
\left\|\tilde{v}_{\text {mult }}\right\|_{\infty}< & \frac{1}{2} \delta t\left(\left\|v_{1}\right\|_{\infty}+\left\|v_{2}\right\|_{\infty}\right)+\frac{1}{2} \delta^{2} t \ell_{w, t B_{\mathrm{key}}} w\left(\left\|v_{1}\right\|_{\infty}+\left\|v_{2}\right\|_{\infty}\right) \\
& +\frac{1}{2} r_{t}(q) \delta^{2} t \ell_{w, t B_{\mathrm{key}}} w+\frac{1}{2} r_{t}(q) \delta t+\frac{1}{2} \delta \min _{i}\left\|v_{i}\right\|_{\infty} \\
& +\frac{1}{2}+r_{t}(q) \delta t+\frac{1}{8}\left(\delta \ell_{w, t B_{\mathrm{key}}} w\right)^{2} .
\end{aligned}
$$

We simplify the expression by replacing the $\left\|v_{i}\right\|_{\infty}$ by a common upper bound $V$, e.g. $V=\max \left\{\left\|v_{1}\right\|_{\infty},\left\|v_{2}\right\|_{\infty}\right\}$. This makes sense if the inherent noise terms are known to be of roughly the same size. If they are of different magnitudes, one gets more precise bounds by using the more complicated formulas that keep these sizes as separate inputs. Using $r_{t}(q)<t$, we obtain the claimed bound.

\section{F Proof of Lemma 3}

It is $f c_{\text {mult }}=\left\langle\tilde{c}_{\text {mult }}, D_{w, q}(f) \otimes D_{w, q}(f)\right\rangle+f\left\langle D_{w, q}\left(\tilde{c}_{\text {mult }}\right), \boldsymbol{e}\right\rangle+g t\left\langle D_{w, q}\left(\tilde{c}_{\text {mult }}\right), s\right\rangle$ $(\bmod q)$. We substitute $\left\langle\tilde{c}_{\text {mult }}, D_{w, q}(f) \otimes D_{w, q}(f)\right\rangle$ from Theorem 1 and obtain $f c=\Delta\left[m_{1} m_{2}\right]_{t}+v_{\text {mult }}(\bmod q)$ with

$$
v_{\text {mult }}=\tilde{v}_{\text {mult }}+f\left\langle D_{w, q}\left(\tilde{c}_{\text {mult }}\right), \boldsymbol{e}\right\rangle+t g\left\langle D_{w, q}\left(\tilde{c}_{\text {mult }}\right), s\right\rangle \quad(\bmod q) .
$$

We bound $\left\|\left\langle D_{w, q}\left(\tilde{c}_{\text {mult }}\right), \boldsymbol{e}\right\rangle\right\|_{\infty} \leq \delta \ell_{w, q}^{3} \frac{w}{2} B_{\text {err }}$. We get the same bound when $\boldsymbol{e}$ is replaced by $s$. With $\|f\|_{\infty},\|t g\|_{\infty}<t B_{\text {key }}$ we deduce $\left\|v_{\text {mult }}\right\|_{\infty}<\left\|\tilde{v}_{\text {mult }}\right\|_{\infty}+$ $2 \delta \cdot t B_{\mathrm{key}} \cdot \delta \ell_{w, q}^{3} \frac{w}{2} B_{\mathrm{err}}$, which proves the lemma. 


\section{G Proof of Theorem 2}

Let $m_{1}, m_{2} \in R$ and let $c_{i}=$ YASHE.Encrypt(pk, $\left.m_{i}\right)$ for $i \in\{1,2\}$. Denote by $v_{i} \in R$ the inherent noise term in $c_{i}$ and suppose $\left\|v_{i}\right\|_{\infty} \leq V<\Delta / 2$. Let $c_{\text {add }}=$ YASHE.Add $\left(c_{1}, c_{2}\right)$ and $c_{\text {mult }}=$ YASHE.Mult $\left(c_{1}, c_{2}, \gamma\right)$ such that $f c_{\text {add }}=$ $\Delta\left[m_{1}+m_{2}\right]_{t}+v_{\text {add }}(\bmod q)$ and $f c_{\text {mult }}=\Delta\left[m_{1} m_{2}\right]_{t}+v_{\text {mult }}(\bmod q)$. Note since $w=2, \ell_{w, q}=\left\lfloor\log _{2}(q)\right\rfloor+2$. Then combining Lemma 3 and Theorem 1 we deduce that

$$
\begin{aligned}
\left\|v_{\text {mult }}\right\|_{\infty}< & \delta t\left(2+\delta \ell_{w, t B_{\mathrm{key}}} w\right) V+\frac{\delta t^{2}}{2}\left(3+\delta \ell_{w, t B_{\mathrm{key}}} w\right)+\frac{1}{8}\left(\delta \ell_{w, t B_{\mathrm{key}}} w\right)^{2}+\frac{1}{2} \\
+ & \delta^{2} t \ell_{w, q}^{3} w B_{\mathrm{err}} B_{\mathrm{key}} .
\end{aligned}
$$

Next, we introduce a common bound $V>0$ for the size of the inherent noise in fresh ciphertexts, and assume that the noise growth for homomorphic additions can be neglected when compared to that for multiplications. By Lemma 2, we know that we can take $V<\delta t B_{\text {key }}\left(2 B_{\text {err }}+\frac{1}{2} r_{t}(q)\right) \leq 2 \delta t^{2} B_{\text {key }} B_{\text {err }}$. By iterating the previous bound for $L$ levels of multiplications, we deduce that for a depth $L$ circuit consisting of additions and multiplications the noise in the ciphertext is bounded by $C_{1}^{L} \cdot V+L C_{1}^{L-1} C_{2}$ where

$$
\begin{gathered}
C_{1}=\delta t\left(2+\delta \ell_{w, t B_{\mathrm{key}}} w\right) \\
C_{2}=\frac{\delta t^{2}}{2}\left(3+\delta \ell_{w, t B_{\mathrm{key}}} w\right)+\frac{1}{8}\left(\delta \ell_{w, t B_{\mathrm{key}}} w\right)^{2}+\frac{1}{2}+\delta^{2} t \ell_{w, q}^{3} w B_{\mathrm{err}} B_{\mathrm{key}}
\end{gathered}
$$

Now observe that $C_{1}=\mathcal{O}(\operatorname{poly}(d) \log (q))$ since $\delta=\phi(d)=d / 2$. Moreover, $C_{2}=\mathcal{O}\left(\operatorname{poly}(d) \log (q)^{3} q^{k}\right)$ and $V=\mathcal{O}\left(\operatorname{poly}(d) \cdot q^{k}\right)$ for some $k \in(1 / 2,1)$. Hence overall, to guarantee correctness, we have that

$$
q=\Omega\left(L \cdot \operatorname{poly}(d)^{L+1} \cdot \log (q)^{L+2} \cdot q^{k}\right) .
$$

The above can be satisfied if

$$
L=\mathcal{O}\left(\frac{(1-k) \log (q)}{\log (\log (q))+\log (d)}\right)
$$

\section{H Fully homomorphic encryption}

The following lemma estimates the depth of the decryption circuit for YASHE.

Lemma 6. The decryption circuit for YASHE can be implemented as a polynomial size circuit of depth $\mathcal{O}(\log (\log (q))+\log (d))$ over $\mathbb{F}_{2}$.

Proof. The first stage of decryption in YASHE consists of the multiplication of two elements of $R_{q}$. In [4, Lemma 4.5], it was shown that this can be computed 
using a circuit of depth $\mathcal{O}(\log (\log (q))+\log (d))$ over $\mathbb{F}_{2}$ (see also [15, Lemma 4.4]). Note that the scaling and rounding operation can be done at a cost of less than the above multiplication with integer multiplications and simple bit shift operations following techniques in [8]. Finally, the reduction modulo 2 does not increase the depth since this simply corresponds to outputting the least significant bit.

Theorem 8 (Fully Homomorphic Encryption). . Under the same assumptions as in Theorem 3 and the additional assumption that YASHE remains IND$C P A$ secure even when an adversary is given encryptions YASHE.Encrypt(pk, sk $[j]$ ) of the bits of the secret key output by YASHE.KeyGen $\left(d, q, 2, \chi_{\mathrm{key}}, \chi_{\mathrm{err}}, 2\right)$, and for the same parameter choices as in Section 4.2, YASHE can be made into an INDCPA fully homomorphic encryption scheme.

Proof. From Theorem 2 we know that YASHE can correctly compute any circuit of depth

$$
\mathcal{O}\left(\frac{(1-k) \log (q)}{\log (\log (q))+\log (d)}\right)=\mathcal{O}\left(\frac{(1-k) d^{\epsilon}}{\log (d)}\right)
$$

for our parameter choices. Since this is greater than the depth of the decryption circuit (for $k, \epsilon$ close to $1 / 2$, say) it follows from Gentry's Bootstrapping Theorem [9] that YASHE can be converted into a fully homomorphic scheme.

\section{Proof of Theorem 4}

Let $v_{1}, v_{2} \in R$ be the inherent noise terms in $c_{1}, c_{2}$. This means we can write $f c_{i}=\Delta\left[m_{i}\right]_{t}+v_{i}+q \cdot r_{i}$ for polynomials $r_{i} \in R$. We assume that the assumptions in Lemma 1 are satisfied and that the $v_{i}$ are chosen such that $\left\|v_{i}\right\|_{\infty}<(\Delta-$ $\left.r_{t}(q)\right) / 2$. In particular, $c_{1}, c_{2}$ are decryptable. The polynomials $r_{i}$ can be bounded by

$$
\left\|r_{i}\right\|_{\infty} \leq \frac{1}{q}\left\|f c_{i}-\Delta\left[m_{i}\right]_{t}-v_{i}\right\|_{\infty}<\frac{1}{q}\left(\delta t B_{\mathrm{key}} \frac{q}{2}+\Delta \frac{t}{2}+\left\|v_{i}\right\|_{\infty}\right) .
$$

Note that we have used the definition of $\delta$ and the bound for the secret key $\|f\|_{\infty}<t B_{\text {key }}$. Since $\Delta \leq q / t$ and $\left\|v_{i}\right\|_{\infty}<\Delta / 2$ we obtain a bound on the $r_{i}$ as $\left\|r_{i}\right\|_{\infty}<\frac{1}{2} \delta t B_{\text {key }}+1$. Multiplying $f c_{1}$ and $f c_{2}$ and substituting the above expression for the $f c_{i}$ yields

$$
\begin{aligned}
f^{2} c_{1} c_{2}= & \Delta^{2}\left[m_{1}\right]_{t}\left[m_{2}\right]_{t}+\Delta\left(\left[m_{1}\right]_{t} v_{2}+\left[m_{2}\right]_{t} v_{1}\right)+q\left(v_{1} r_{2}+r_{1} v_{2}\right)+ \\
& v_{1} v_{2}+q \Delta\left(\left[m_{1}\right]_{t} r_{2}+r_{1}\left[m_{2}\right]_{t}\right)+q^{2} r_{1} r_{2} .
\end{aligned}
$$

Lemma 5 in Appendix D incorporates $\left[m_{1} m_{2}\right]_{t}$ and scales by $t / q$, i.e. it gives us an expression for $\frac{t}{q} f^{2} c_{1} c_{2}$ which contains $\Delta\left[m_{1} m_{2}\right]_{t}$ as a summand. But to 
bound the size of the inherent noise term in $\tilde{c}_{\text {mult }}$, we need to consider $f^{2} \tilde{c}_{\text {mult }} \equiv$ $f^{2}\left\lfloor\frac{t}{q} c_{1} c_{2}\right\rceil(\bmod q)$. We consider the difference

$$
r_{a}=\frac{t}{q} f^{2} c_{1} c_{2}-f^{2}\left\lfloor\frac{t}{q} c_{1} c_{2}\right\rceil \equiv \frac{t}{q} f^{2} c_{1} c_{2}-f^{2} \tilde{c}_{\text {mult }} \quad(\bmod q)
$$

This difference can be bounded by

$$
\left\|r_{a}\right\|_{\infty}=\left\|f^{2}\left(\frac{t}{q} c_{1} c_{2}-\left\lfloor\frac{t}{q} c_{1} c_{2}\right\rceil\right)\right\|_{\infty} \leq \frac{1}{2} \delta^{2}\|f\|_{\infty}^{2} \leq \frac{1}{2}\left(\delta t B_{\mathrm{key}}\right)^{2} .
$$

In the following lemma, we bound the inherent noise term in the intermediate ciphertext $\tilde{c}_{\text {mult }}$ after the first part of the homomorphic multiplication procedure. This is very similar to [8]. To prove Theorem 4, we simplify the expression for the bound obtained in Lemma 7 by replacing the $\left\|v_{i}\right\|_{\infty}$ by the common upper bound $V$. Using $r_{t}(q)<t$, we obtain the bound in Theorem 4 .

Lemma 7. Let $c_{1}, c_{2} \in R$ be ciphertexts encrypting $m_{1}, m_{2} \in R$, which are decryptable with the secret key $f$. Assume that the inherent noise terms $v_{1}, v_{2} \in R$ in $c_{1}, c_{2}$ satisfy $\left\|v_{i}\right\|_{\infty}<\Delta / 2$. Let $f=1+t f^{\prime}$ with $f^{\prime} \leftarrow \chi_{\text {key }}$ where $\left\|\chi_{\text {key }}\right\|_{\infty}<$ $B_{\text {key }}$. Define

$$
\tilde{c}_{\text {mult }}=\left[\left\lfloor\frac{t}{q} \cdot c_{1} c_{2}\right\rceil\right]_{q}
$$

Then $f^{2} \tilde{c}_{\text {mult }}=\Delta\left[m_{1} m_{2}\right]_{t}+\tilde{v}_{\text {mult }}(\bmod q)$ where

$$
\begin{aligned}
\left\|\tilde{v}_{\text {mult }}\right\|_{\infty}< & \frac{1}{2}\left(\delta t\left(3+\delta t B_{\text {key }}\right)\left(\left\|v_{1}\right\|_{\infty}+\left\|v_{2}\right\|_{\infty}\right)+\delta \min _{i}\left\|v_{i}\right\|_{\infty}\right. \\
& \left.+\delta t r_{t}(q)\left(5+\delta t B_{\text {key }}\right)+\left(\delta t B_{\text {key }}\right)^{2}+1\right) .
\end{aligned}
$$

Proof. Equation (5) and Lemma 5 yield $f^{2} \tilde{c}_{\text {mult }}=\frac{t}{q} f^{2} c_{1} c_{2}-r_{a}=\Delta\left[m_{1} m_{2}\right]_{t}+$ $\tilde{v}_{\text {mult }}(\bmod q)$, where we define

$\tilde{v}_{\text {mult }}=\left[m_{1}\right]_{t} v_{2}+\left[m_{2}\right]_{t} v_{1}+t\left(v_{1} r_{2}+r_{1} v_{2}\right)-r_{t}(q)\left(\left[m_{1}\right]_{t} r_{2}+\left[m_{2}\right]_{t} r_{1}+r_{m}\right)+r_{v}+r_{r}-r_{a}$

It follows

$$
\begin{aligned}
\left\|\tilde{v}_{\text {mult }}\right\|_{\infty} \leq & \left\|\left[m_{1}\right]_{t} v_{2}+\left[m_{2}\right]_{t} v_{1}\right\|_{\infty}+t\left\|v_{1} r_{2}+r_{1} v_{2}\right\|_{\infty}+r_{t}(q)\left\|\left[m_{1}\right]_{t} r_{2}+\left[m_{2}\right]_{t} r_{1}\right\|_{\infty} \\
& +r_{t}(q)\left\|r_{m}\right\|_{\infty}+\left\|r_{v}\right\|_{\infty}+\left\|r_{r}\right\|_{\infty}+\left\|r_{a}\right\|_{\infty} .
\end{aligned}
$$

We bound the summands separately and add together to obtain the overall bound. We have $\left\|\left[m_{1}\right]_{t} v_{2}+\left[m_{2}\right]_{t} v_{1}\right\|_{\infty} \leq \delta \frac{t}{2}\left(\left\|v_{1}\right\|_{\infty}+\left\|v_{2}\right\|_{\infty}\right)$, since the coefficients of the $\left[m_{i}\right]_{t}$ are of absolute value at most $t / 2$. Similarly, the bounds on $\left\|r_{i}\right\|_{\infty}$ lead to $\left\|v_{1} r_{2}+r_{1} v_{2}\right\|_{\infty} \leq \delta \cdot\left(\frac{1}{2} \delta t B_{\text {key }}+1\right)\left(\left\|v_{1}\right\|_{\infty}+\left\|v_{2}\right\|_{\infty}\right)$, and $\left\|\left[m_{1}\right]_{t} r_{2}+\left[m_{2}\right]_{t} r_{1}\right\|_{\infty} \leq \delta t\left(\frac{1}{2} \delta t B_{\text {key }}+1\right)$. We have already obtained bounds for 
$\left\|r_{m}\right\|_{\infty},\left\|r_{v}\right\|_{\infty},\left\|r_{r}\right\|_{\infty}$, and $\left\|r_{a}\right\|_{\infty}$ above. We summarize and get

$$
\begin{aligned}
\left\|\tilde{v}_{\text {mult }}\right\|_{\infty}< & \frac{1}{2} \delta t\left(\left\|v_{1}\right\|_{\infty}+\left\|v_{2}\right\|_{\infty}\right)+\delta t\left(\frac{1}{2} \delta t B_{\text {key }}+1\right)\left(\left\|v_{1}\right\|_{\infty}+\left\|v_{2}\right\|_{\infty}\right) \\
& +r_{t}(q) \delta t\left(\frac{1}{2} \delta t B_{\text {key }}+1\right)+\frac{1}{2} r_{t}(q) \delta t+\frac{1}{2} \delta \min _{i}\left\|v_{i}\right\|_{\infty} \\
& +\frac{1}{2}+r_{t}(q) \delta t+\frac{1}{2}\left(\delta t B_{\text {key }}\right)^{2} .
\end{aligned}
$$

A rearrangement of this expression yields the claimed bound for $\left\|\tilde{v}_{\text {mult }}\right\|_{\infty}$.

\section{J Proof of Lemma 4}

It is $f c=f\left\langle D_{w, q}\left(\tilde{c}_{\text {mult }}\right), \gamma\right\rangle=f\left\langle D_{w, q}\left(\tilde{c}_{\text {mult }}\right), P_{w, q}(f)+\boldsymbol{e}+h \boldsymbol{s}\right\rangle=f^{2} \tilde{c}_{\text {mult }}+$ $f\left\langle D_{w, q}\left(\tilde{c}_{\text {mult }}\right), \boldsymbol{e}\right\rangle+\operatorname{tg}\left\langle D_{w, q}\left(\tilde{c}_{\text {mult }}\right), \boldsymbol{s}\right\rangle(\bmod q)$. We use $f^{2} \tilde{c}_{\text {mult }}=\Delta\left[m_{1} m_{2}\right]_{t}+$ $\tilde{v}_{\text {mult }}$ and obtain $f c=\Delta\left[m_{1} m_{2}\right]_{t}+v_{\text {mult }}(\bmod q)$ with

$$
v_{\text {mult }}=\tilde{v}_{\text {mult }}+f\left\langle D_{w, q}\left(\tilde{c}_{\text {mult }}\right), \boldsymbol{e}\right\rangle+t g\left\langle D_{w, q}\left(\tilde{c}_{\text {mult }}\right), s\right\rangle \quad(\bmod q) .
$$

We bound $\left\|\left\langle D_{w, q}\left(\tilde{c}_{\text {mult }}\right), \boldsymbol{e}\right\rangle\right\|_{\infty}=\left\|\sum_{i=0}^{\ell_{w, q}-1}\left[\tilde{c}_{i}\right]_{w} e_{i}\right\|_{\infty} \leq \delta \ell_{w, q} \frac{w}{2} B_{\text {err }}$, where $\tilde{c}_{\text {mult }}=\sum\left[\tilde{c}_{i}\right]_{w} w^{i}$ and $\boldsymbol{e}=\left(e_{i}\right)$. We get the same bound when $\boldsymbol{e}$ is replaced by $s$. With $\|f\|_{\infty},\|t g\|_{\infty}<t B_{\text {key }}$ we deduce $\left\|v_{\text {mult }}\right\|_{\infty}<\left\|\tilde{v}_{\text {mult }}\right\|_{\infty}+2 \delta \cdot t B_{\text {key }}$. $\delta \ell_{w, q} \frac{w}{2} B_{\text {err }}$, which proves the lemma.

\section{K Detailed Noise Bounds for YASHE'}

The following lemma summarizes detailed bounds on the noise growth during homomorphic addition and multiplication operations. These bounds are more detailed than the ones given in Section 5.1 and depend on the individual inherent noise sizes $\left\|v_{i}\right\|_{\infty}$ in each of the ciphertexts. Depending on the exact computation that is to be done, these bounds might be more accurate and might lead to more efficient parameters than the more coarse bounds given in Section 5.1.

Lemma 8. Let (pk, sk, evk) $=(h, f, \gamma) \in R^{2}$ be output by YASHE'.KeyGen. Let $m_{1}, m_{2} \in R$ and let $c_{i}=\mathrm{YASHE}^{\prime}$.Encrypt $\left(\mathrm{pk}, m_{i}\right)$ for $i \in\{1,2\}$. Denote by $v_{i} \in R$ the inherent noise term in $c_{i}$ and suppose $\left\|v_{i}\right\|_{\infty}<\Delta / 2$. Let $c_{\text {add }}=$ $\mathrm{YASHE}^{\prime} \cdot \operatorname{Add}\left(c_{1}, c_{2}\right)$ and $c_{\mathrm{mult}}=\mathrm{YASHE}^{\prime} \cdot \operatorname{Mult}\left(c_{1}, c_{2}, \gamma\right)$ be such that $f c_{\text {add }}=$ $\Delta\left[m_{1}+m_{2}\right]_{t}+v_{\text {add }}(\bmod q)$ and $f c_{\text {mult }}=\Delta\left[m_{1} m_{2}\right]_{t}+v_{\text {mult }}(\bmod q)$. Let $w>1$ be an integer and let $\ell_{w, q}=\left\lceil\log _{w}(q)\right\rceil+2$. Then

$$
\begin{aligned}
\left\|v_{\text {add }}\right\|_{\infty} \leq & \left\|v_{1}\right\|_{\infty}+\left\|v_{2}\right\|_{\infty}+r_{t}(q) \\
\left\|v_{\text {mult }}\right\|_{\infty}< & \frac{1}{2}\left(\delta t\left(3+\delta t B_{\text {key }}\right)\left(\left\|v_{1}\right\|_{\infty}+\left\|v_{2}\right\|_{\infty}\right)+\delta \min _{i}\left\|v_{i}\right\|_{\infty}\right. \\
& \left.+\delta t_{t}(q)\left(5+\delta t B_{\text {key }}\right)+\left(\delta t B_{\text {key }}\right)^{2}+1\right)+\delta^{2} t \ell_{w, q} w B_{\text {err }} B_{\text {key }} .
\end{aligned}
$$

Proof. This is a combination of Inequality (1) and Lemmas 7 and 4. 


\section{Proof of Theorem 5}

The proof is subdivided into the following corollary and lemma. The corollary simply combines the noise growth bounds from the first step of the multiplication and from the key switching part based on a common upper bound $V$ for the inherent noise of the original ciphertexts.

Corollary 1. In addition to the assumptions made in Lemma 8, let $V>0$ be such that $\left\|v_{i}\right\|_{\infty} \leq V<\Delta / 2$ for $i \in\{1,2\}$. Let $\epsilon_{1}=4\left(\delta t B_{\mathrm{key}}\right)^{-1}$. Then the norms of the inherent noise terms $v_{\text {add }}$ and $v_{\text {mult }}$ satisfy $\left\|v_{\text {add }}\right\|_{\infty} \leq 2 V+r_{t}(q)<$ $2 V+t$ and $\left\|v_{\text {mult }}\right\|_{\infty}<C_{1} V+C_{2}$, for

$$
C_{1}=\left(1+\epsilon_{1}\right) \delta^{2} t^{2} B_{\mathrm{key}}, \quad C_{2}=\delta^{2} t B_{\mathrm{key}}\left(t\left(B_{\mathrm{key}}+t\right)+\ell_{w, q} w B_{\mathrm{err}}\right) .
$$

Proof. Theorem 4 shows that we can take $C_{1}=\delta t\left(4+\delta t B_{\text {key }}\right)$, which is equal to the above expression. We get the constant $C_{2}$ from Theorem 4 and Lemma 4 .

The following lemma iterates $L$ levels of multiplications and deduces an overall noise bound for this operation.

Lemma 9. Let $c \in R$ be a ciphertext that is the homomorphic product of ciphertexts of inherent noise size at most $V$ arranged in a tree of $L$ levels of multiplications. Let $v \in R$ be the inherent noise term in $c$. Then the norm of $v$ can be bounded by

$$
\|v\|_{\infty}<C_{1}^{L} V+L C_{1}^{L-1} C_{2} .
$$

Using the bounds in Corollary 1, we obtain

$$
\begin{aligned}
\|v\|_{\infty}< & \left(1+\epsilon_{1}\right)^{L}(\delta t)^{2 L} B_{\mathrm{key}}^{L} V \\
& +L\left(1+\epsilon_{1}\right)^{L-1} \delta^{2 L} t^{2 L-1} B_{\mathrm{key}}^{L}\left(t\left(B_{\mathrm{key}}+t\right)+\ell_{w, q} w B_{\mathrm{err}}\right) \\
= & \left(1+\epsilon_{1}\right)^{L-1} \delta^{2 L} t^{2 L-1} B_{\mathrm{key}}^{L}\left(\left(1+\epsilon_{1}\right) t V+L\left(t\left(B_{\mathrm{key}}+t\right)+\ell_{w, q} w B_{\mathrm{err}}\right)\right) .
\end{aligned}
$$

Proof. This follows by induction on $L$ by repeatedly applying the bound in Corollary 1.

Theorem 5 follows from Lemma 9 and Lemma 1. 